\title{
Psychomotorische Defizite von Kindern im Grundschulalter und ihre Auswirkungen auf die Radfahr-Ausbildung
}

Dr. Rudolf Günther

Dipl.-Ing. Sabine Degener 


\title{
Psychomotorische Defizite von Kindern im Grundschulalter und ihre Auswirkungen auf die Radfahr-Ausbildung
}

\author{
Dr. Rudolf Günther \\ Dipl.-Ing. Sabine Degener
}

Unfallforschung der Versicherer 
Die Unfallforschung der Versicherer veröffentlicht ihre Forschungsergebnisse in den Reihen:

FS - Fahrzeugsicherheit

VI - Verkehrsinfrasturktur

VV - Verkehrsverhalten / Verkehrspädagogik

\section{Impressum:}

Gesamtverband der Deutschen Versicherungswirtschaft e. V.

\section{Unfallforschung der Versicherer}

Wilhelmstraße 43 / 43G, 10117 Berlin

Postfach 0802 64, 10002 Berlin

E-Mail: unfallforschung@gdv.org

Internet: www.udv.de

ISBN-Nr.: 978-3-939163-24-4

Erschienen: 2009

Redaktion: Klaus Brandenstein

Layout: Franziska Gerson Pereira

Technik: Wilfried Butenhof 


\section{Psychomotorische Defizite von Kindern im Grundschulalter und ihre Auswirkungen auf die Radfahr-Ausbildung}

bearbeitet durch

Prof. Doz. Dr. Rudolf Günther unter Mitarbeit von cand. psych. Anja Diez Reutlingen 2009 Forschungsgruppe Dr. Günther

Fachliche Betreuung:

Dipl.-Ing. Sabine Degener, UDV Martin Kraft, VMS 


\section{Vorbemerkung}

Viele Kinder, vor allem im großstädtischen Bereich, haben im Vergleich zum Jahr 1997 deutliche psychomotorische Schwächen. Das wird besonders bei der Fahrradausbildung sichtbar, die nahezu alle Grundschüler in 4. Klasse absolvieren. Die aktuelle Studie der Unfallforschung der Versicherer (UDV) beleuchtet die Fähig- und Fertigkeiten von Kindern im Grundschulalter und deren Auswirkung auf die Fahrradausbildung. Darin wird deutlich, dass vor allem Stadtkinder, Mädchen mit Migrationshintergrund, übergewichtige und überbehütete Kinder auffallen. Um den Teufelskreis „Fehlende Bewegung - mehr Unsicherheit - weniger Fahrradpraxis - weniger Bewegung" zu durchbrechen, müssten Schulen, Eltern und Lehrer gezielt Bewegungsdefizite erkennen und bekämpfen.

\section{Radfahrausbildung}

Rund 95 \% aller Viertklässler legen pro Jahr die Fahrradprüfung ab. Einerseits ist die Radfahrausbildung ein wichtiger Teil der Mobilitäts- und Verkehrserziehung, anderseits werden durch sie auch psychomotorische Mängel schonungslos aufgedeckt. So beherrschen immer mehr Kinder wichtige Alltagssituationen des Radfahrens nicht, beispielsweise das Spurhalten beim Blick zur Seite oder nach hinten. Diese Defizite, die vor allem in Großstädten sichtbar werden, können während der Fahrradausbildung aber immer seltener ausgeglichen werden. So konnten 2008 nur noch halb so viele Einzelförderungen und Nachkurse durchgeführt werden wie 1997. Dabei ist die Radfahrausbildung in den vergangenen Jahren deutlich praxisnäher geworden. Es werden nicht nur „Trockenübungen“ auf abgesperrten Plätzen gemacht, sondern immer öfter wird ein Teil der Ausbildung im realen Straßenverkehr absolviert.

\section{Jugendverkehrsschulen}

Rund 1.100 Jugendverkehrsschulen gibt es in Deutschland. Sie sind ein maßgeblicher Träger der Fahrradausbildung. Deutlich abgenommen hat in den vergangenen Jahren die Rolle der festen Plätze gegenüber den „mobilen“ Jugendverkehrsschulen, die auch vor Ort, z. B. in den Schulhöfen, Angebote zur Fahrradausbildung machen können. Die Jugendverkehrsschulen werden von viel ehrenamtlichem Engagement getragen, sind aber oft mit nur geringen finanziellen Mitteln ausgestattet. Um diese wichtige Arbeit der Verkehrs- und Mobilitätserziehung zu unterstützen, stellt die Unfallforschung der Versicherer jährlich 100.000 Euro zur Verfügung, die über die Verkehrswachten an die Jugendverkehrsschulen - vorwiegend für Reparaturmaßnahmen und Fahrradneuanschaffungen - verteilt werden. 


\section{Kinder als Radfahrer}

Für viele Kinder hat die eigenständige Mobilität in den vergangenen Jahren abgenommen. Sie werden vermehrt mit dem Auto zur Schule gefahren oder müssen in ländlichen Gebieten auch schon früh mit dem Bus zur Schule fahren. Das Fahrrad ist für Kinder zwar immer noch wichtiger Garant für eigenständige Mobilität. „Stubenhockern“ fehlt aber die Praxis und macht das gelegentliche Radfahren dann umso gefährlicher. Deshalb sollten vor allem die Eltern in der Freizeit und an Wochenenden oder in den Ferien ausgiebige Radtouren mit ihren Kindern unternehmen, um deren Fähigkeiten, sich sicher auf dem Zweirad im Straßenverkehr zu bewegen, zu fördern.

\section{Was muss getan werden?}

- Kinder brauchen viel Bewegung. Diese kann zu Hause, in der Schule und im Sportverein gefördert werden.

- Grundschulen müssen den Raum und die Zeit bieten, Mobilitätsdefizite von Kindern zu erkennen und auszugleichen.

- Eltern sollten ihre Kinder nicht mit dem Auto zur Schule bringen, sondern zu Fuß.

- Während der Fahrradausbildung sollte es genug Zeit für Einzelförderung und Nachschulungen geben.

- Für den sicheren Schulweg mit dem Fahrrad ab der Klasse 5 sollten Schulwegpläne erstellt und ernst genommen werden (siehe auch www.udv.de). 


\section{Inhalt}

$\begin{array}{ll}\text { Vorbemerkung } & 4\end{array}$

$\begin{array}{lll}1 & \text { Problemstellung } & 7\end{array}$

$\begin{array}{lll}2 & \text { Basisbefunde } & 7\end{array}$

2.1 Stichproben- Kenngrößen 7

2.2 Regionalverteilung 8

2.3 Hinweisezu Selbstselektionseffekten für die Rücklaufquote 9

2.4 Urbanisierungsgrad des Regionalumfeldes 9

$\begin{array}{lll}3 & \text { Hauptergebnisse } & 10\end{array}$

3.1 Umsetzungsrahmen der Radfahrausbildung in Deutschland $\quad 10$

3.2 Motorische Schwächen von Kindern 16

3.3 Stellenwert von motorischen Schwierigkeiten für die Anforderungsbewältigung in der Radfahrausbildung 23

3.4 Organisatorische Maßnahmen zur Sicherung der Kursdurchführung 25

4 Diskussion der Hauptergebnisse und Zusammenfassung 30

4.1 Stichprobenmerkmale 30

4.2 Umsetzungsrahmen der Radfahrausbildung in Deutschland 31

4.3 Motorische Schwächen von Kindern 31

4.4 Organisatorische Maßnahmen zur Sicherung
der Kursdurchführung

4.5 Allgemeinere Problemperspektiven 33

$\begin{array}{ll}\text { Literatur } & 34\end{array}$

$\begin{array}{ll}\text { Anlage } & 35\end{array}$ 


\section{Problemstellung}

Zur Einordnung der hier bearbeiteten Fragestellungen sollte zunächst Beachtung finden, dass in der aktuellen verkehrswissenschaftlichen Diskussion in Deutschland Fragen der Radfahrsicherheit und dabei insbesondere der Radfahrausbildung einen wichtigen Stellenwert einnehmen (vgl. [2]). In diesem Zusammenhang bilden ausreichende Körperbeherrschung und psychomotorische Fertigkeiten der Kinder eine elementare Voraussetzung auch für die Teilnahme an der Radfahrausbildung gegen Ende der Grundschulzeit. Fachkräfte der Polizei-Verkehrserziehungsdienste und verkehrspädagogisch aktive Lehrer/-innen haben daher zugleich besonders umfangreiche Möglichkeiten, die Bewegungsgeschicklichkeit von Kindern am Ende der Grundschulzeit zu beobachten; zudem sollte sich ein Rückgang motorischer Fertigkeiten unmittelbar auf die Durchführung der entsprechenden Ausbildungskurse auswirken. Die hier vorgelegte Studie befasst sich dazu mit zwei Fragenbereichen:

(1) Da über den Verbreitungsgrad von psychomotorischen Defiziten von Kindern im Kleinkind- und Grundschulalter und vor allem über eine mögliche Zunahme von Bewegungsmängeln derzeit lediglich regional begrenzte Befunde (vgl. [1]) zur Verfügung stehen, können durch eine bundesweite Befragung dieser praxisnah tätigen Fachkräfte wichtige inhaltliche Hinweise über den bestehenden Problemumfang erwartet werden. Durch die (recht kurze) Erhebung sollten dabei folgende Problembereiche näher abgeklärt werden:

- Von welchen Basiskenngrößen ist derzeit bei der Umsetzung der Radfahrausbildung in Deutschland auszugehen?
- Was lässt sich zum Problemprofil der motorischen Schwächen von Kindern aussagen (Verbreitungsgrad und Veränderungstrend, Ursachen für Problemschwerpunkte)?

- Welcher Stellenwert kommt motorischen Schwierigkeiten der Kinder im Aufgabenspektrum der Radfahrausbildung zu (Schwierigkeiten bei Bewältigung von grundlegenden Anforderungen vs. Beeinträchtigung beim Beherrschen anspruchsvoller, schwieriger Programmteile)?

- Auf welche organisatorische Maßnahmen wird zur Sicherung der Kursdurchführung zurückgegriffen?

(2) Da andererseits die aktuell - zum Jahreswechsel 2008/09 - bundesweit durchgeführte Befragung eine inhaltlich weitgehende Wiederholung einer bereits vor etwa 10 Jahren im Auftrag der Deutschen Verkehrswacht durchgeführten Befragung (vgl. [4]) bildet, können zu den heute erhobenen Einzelthemen empirisch gestützte Einschätzungen über Umfang und Geschwindigkeit von Veränderungsprozessen abgeleitet werden und damit zugleich eine grobe Vorschau auf die zukünftige Problementwicklung.

Aussagen zu Veränderungen sind im Text kursiv hervorgehoben.

\section{Basisbefunde}

\subsection{Stichproben- Kenngrößen}

\section{Datengrundlage}

Grundlage der Studie bildet eine schriftliche Anfrage bei $n=1008$ Verkehrserziehungs-Dienststellen (Polizei, Schulen) aufgrund einer Adress- 
Datei der Deutschen Verkehrswacht mit weiter Streuung über das gesamte Bundesgebiet.

Der Fragebogenversand erfolgte an der Jahreswende 2008/09. Aufgrund des Rücklaufes stehen insgesamt $n=368$ Fragebögen - entsprechend einer Rücklaufquote von 36,5 \% - und für die Datenanalyse $n=347$ vollständig bearbeitete Fragebogen zur Verfügung.

Die 1997 erreichte, sehr hohe Rücklaufquote (74,9\% entspicht $n=217$ von einer wesentlich geringeren Versandstichprobe $(n=246))$ konnte damit allerdings nicht mehr erzielt werden.

Obwohl die Befragung in anonymer Form durchgeführt wurde, erfolgte eine zusätzliche, freiwillig erbetene Angabe zur beantwortenden Dienststelle oder Person in beachtlicher Höhe von 79 \% der Fälle (gegenüber 1997 mit extrem hoher Quote von $90 \%$ ).
Ein nicht ganz unerheblicher Anteil der Befragungen wurde dabei nicht durch Dienststellen der Polizei, sondern von Schulen beantwortet, dies könnte auf systematische Unterschiede in der organisatorischen Durchführung der Radfahrausbildung - regional und nicht zuletzt in unterschiedlichen Bundesländern - verweisen; vertiefende Analysen hierzu finden sich später unter 3.1.

\subsection{Regionalverteilung}

Einen Überblick über die Regionalverteilung der Untersuchungsstichprobe gibt die folgende Grafik (Zugehörigkeit zum Bundesland wurde 1997 nicht erhoben, Vergleichsdaten aus der Erstbefragung stehen daher nicht zur Verfügung, vgl. aber grobe Hinweise zu Postleitregionen (siehe Grafik 2)).

Am höchsten sind die Teilstichproben - mit hohem Abstand - aus Bayern (34\%), gefolgt von

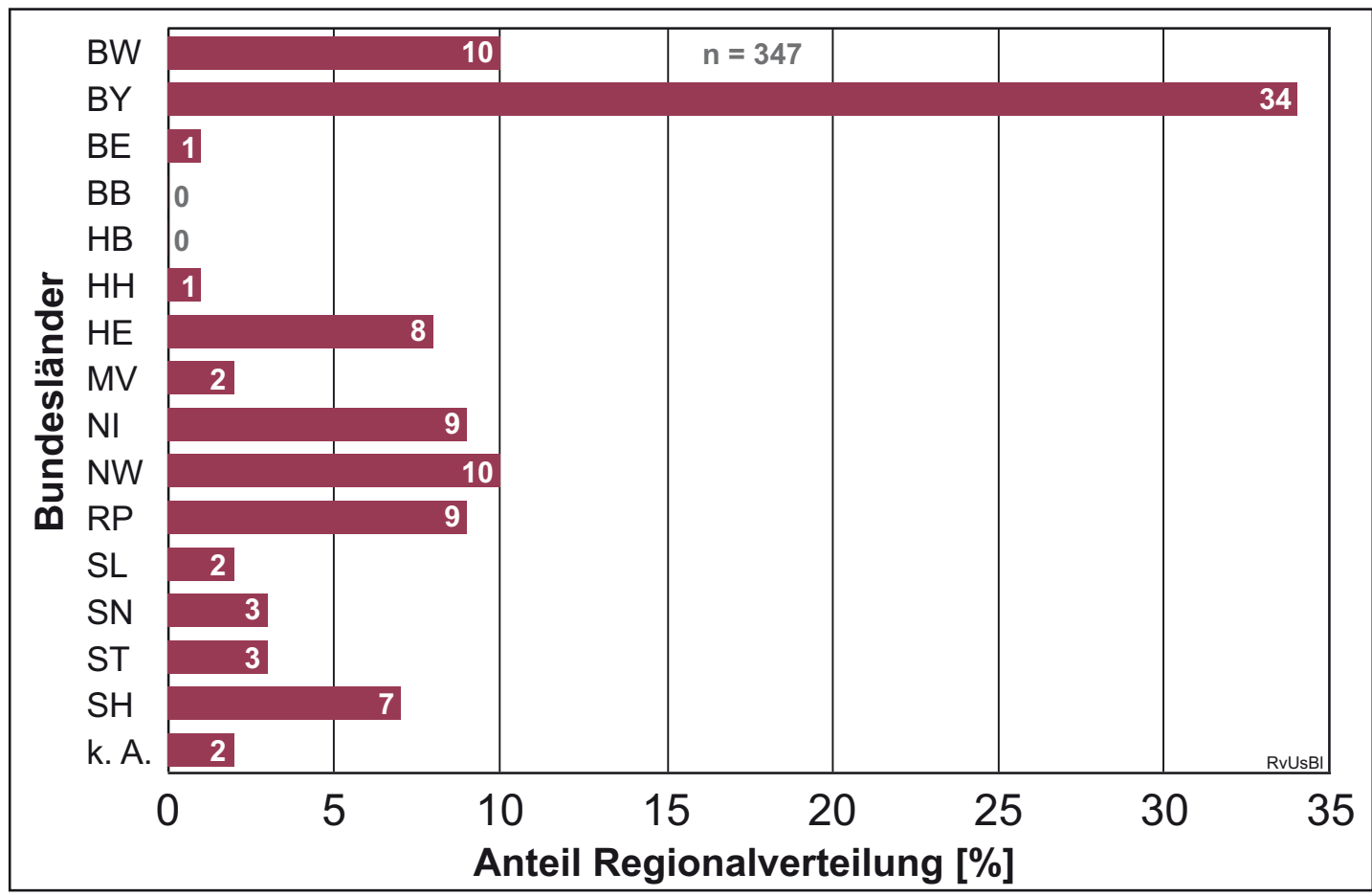

Grafik 1: Regionalverteilung der Untersuchungsstichprobe nach Bundesländern (in Prozent, $n=347$ ) 
Nordrhein-Westfalen und Baden-Württemberg (je $10 \%)$, Niedersachsen und RheinlandPfalz (9\%), Hessen (8\%), Schleswig-Holstein (7\%).

\subsection{Hinweise zu Selbstselektionseffekten für die Rücklaufquote}

Daten über möglicherweise unterschiedliche Rücklaufquoten in den einzelnen Bundesländern bei Beteiligung an der Befragung standen nicht zur Verfügung. Vielmehr stützt sich die Analyse auf die freiwillig erbetenen Angaben der Postleitregion der beantwortenden Dienststelle - hierzu liegen direkte Angaben von $n=136$ Fragebögen, entsprechend 39,2\% der bearbeiteten Bögen vor -, die mit der entsprechenden Regionalverteilung in der Versandstichprobe verglichen wird. Eine grobe Abschätzung der - möglicherweise regional unterschiedlichen - Rücklaufquote gibt die folgende Grafik, deren Aussagekraft allerdings eingeschränkt ist.

Ersichtlich ist zunächst (vgl. vorausgegangene Grafik), dass die Versandstichprobe relative Regionalschwerpunkte in den PL-Regionen 2/ Hamburg, 5/Köln, 8/München und 9/Nürnberg aufweist.

Die wichtigen Unterschiede zwischen Versand- und Rücklaufprofil konzentrieren sich (auch gemäß statistischer (Chi-Quadrat-) Prüfgröße) auf diese vier Schwerpunktregionen: Eine quantitativ deutlich verminderte Beteiligung an der Befragung ist in zwei der vier dieser Schwerpunktregionen zu konstatieren, nämlich aus den PL- Regionen 2/Hamburg, Schleswig-Holstein und 5/Köln, während die beiden südlichen Schwerpunktregionen in Bayern (mit Thüringen) recht klar erhöhte Rücklaufquoten aufweisen.

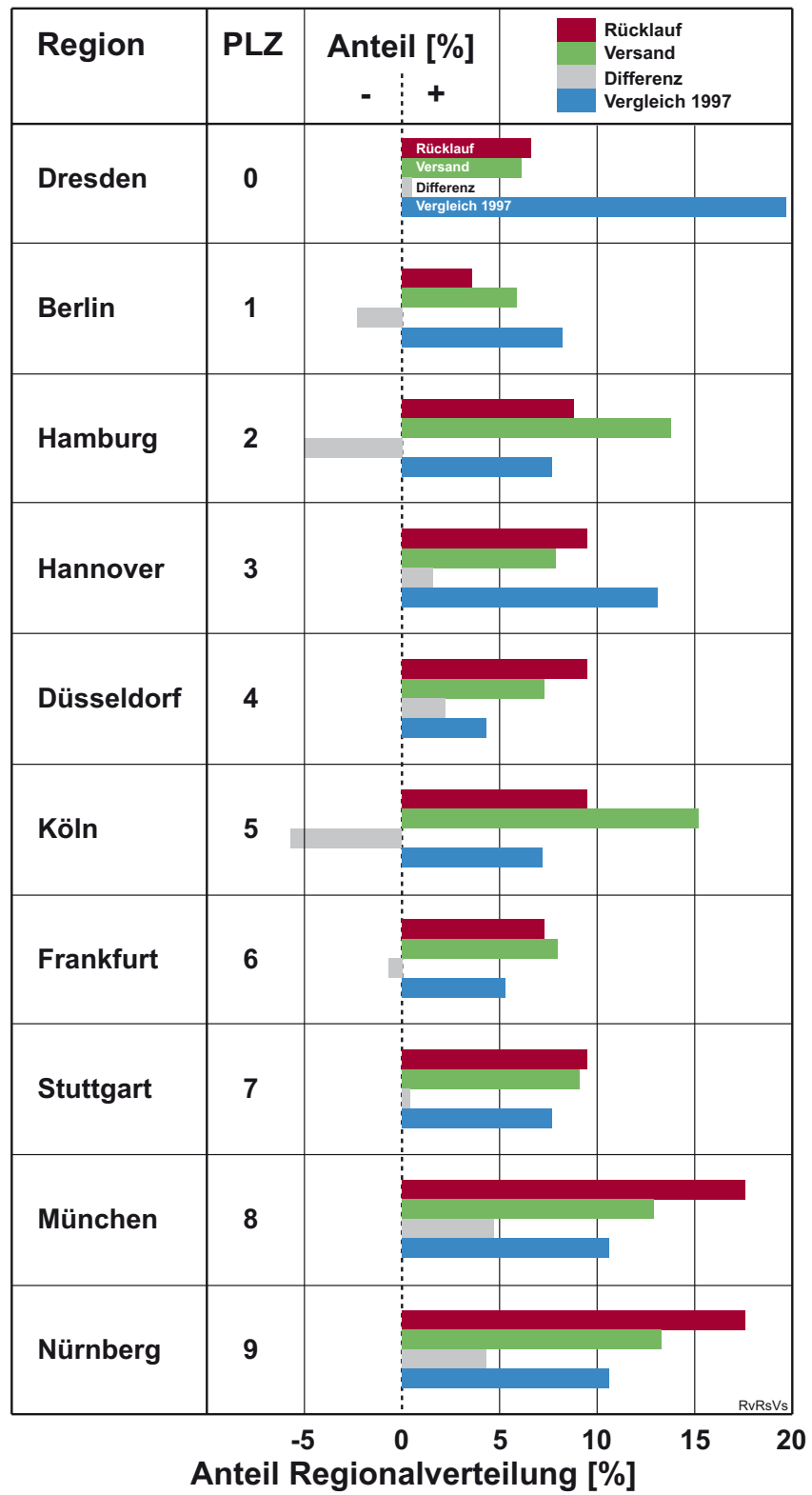

Grafik 2: Regionalverteilung der Rücklaufstichprobe im Vergleich zur Regionalverteilung der Versandstichprobe (Postleitregionen); untere Zeile: Vergleichswerte Rücklauf 1997 (\%)

\subsection{Urbanisierungsgrad des Regionalumfeldes}

Das regionale Einzugsgebiet der einbezogenen Schulklassen in der Gesamtstichprobe wird von den Befragten wie folgt beschrieben (vgl. Grafik 3): 


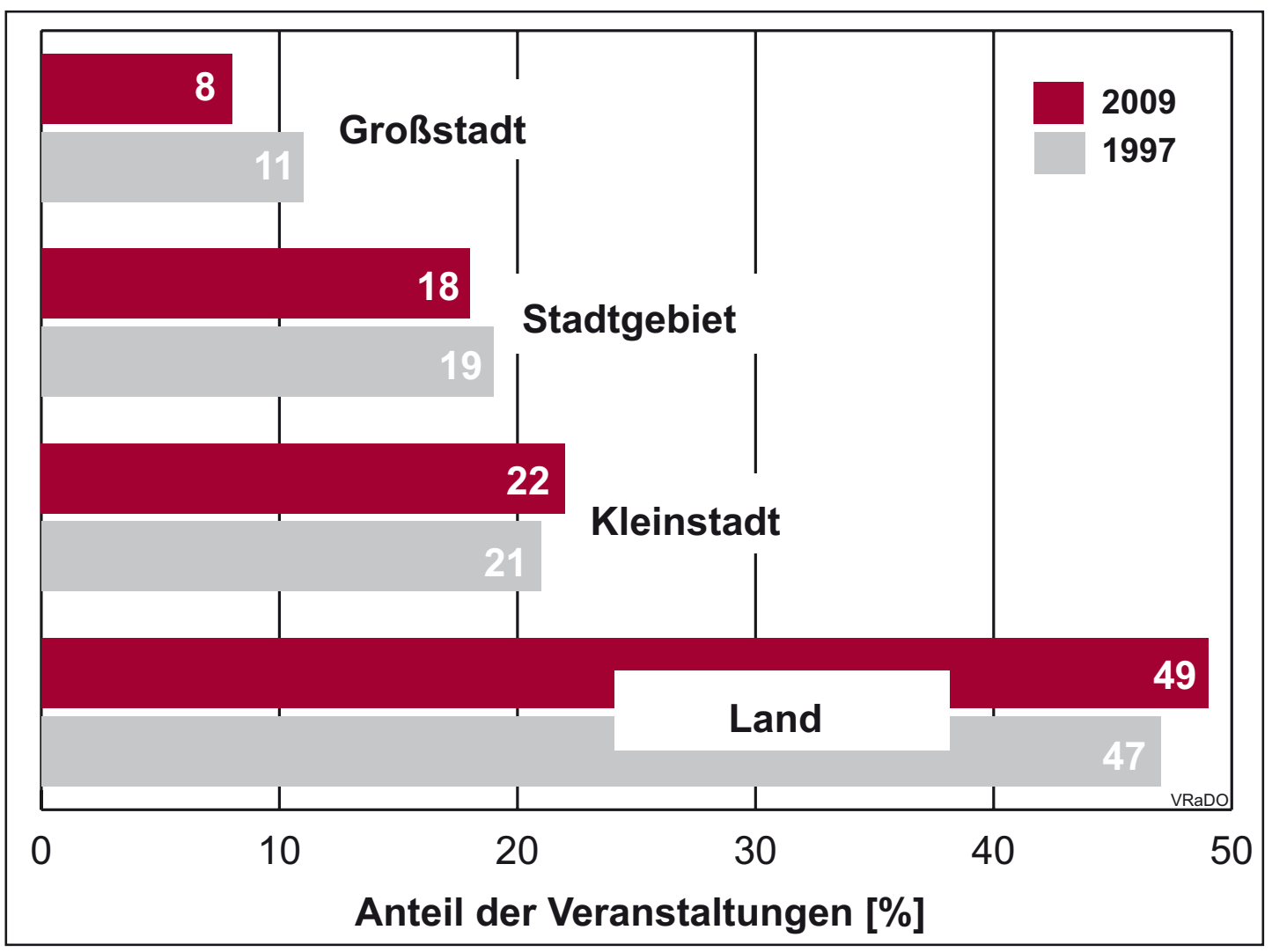

Grafik 3: Anteil der durchgeführten Veranstaltungen zur Radfahrausbildung bei Differenzierung nach Ortstypen

Innerhalb der Untersuchungsstichprobe findet sich also ein deutlicher Schwerpunkt der durchgeführten Radfahrveranstaltungen in dörflichen und ländlichen Gebieten. Gegenüber der Ersterhebung 1997 finden sich dabei nahezu keine Verschiebungen.

\section{Hauptergebnisse}

\subsection{Umsetzungsrahmen der Radfahrausbildung in Deutschland}

\section{Reichweite}

Von den an der Befragung teilnehmenden Dienststellen wurden im zurückliegenden
Schuljahr 204.638 Kinder aus mehr als 13.460 Schulklassen erreicht, im Durchschnitt 40,2 Klassen pro Rückmeldung.

Vergleich zur Ersterhebung 1997: Zwar muss bei wesentlichen Kennziffern für den Vergleichszeitraum 1997 ein Rückgang konstatiert werden (mit einem Durchschnitt von 2066 Kindern aus mehr als 55 Schulklassen pro Erhebungseinheit und 423.587 Kindern, die an der Radfahr-Ausbildung teilgenommen hatten), die Unterschiede dürften aber vorrangig durch externe Randbedingungen - vor allem auch die organisatorische Umsetzung der Radfahrausbildung durch Schulen, möglicherweise auch die Schülerzahl/Klasse - erklärbar sein. (Methodische Anmerkung: Die Befundlage kann insoweit verzerrt sein, als auch Fragebogenbearbeitungen enthalten sind, die sich auf die Zahl der Klassen pro Schule beziehen (siehe 3.1).) 


\section{Flächendeckende Regionalumsetzung}

In immerhin 85,7 \% der teilnehmenden Erhebungseinheiten wurden 2008/09 alle vorhandenen Schulklassen komplett erreicht (keine Daten aus der Ersterhebung 1997).

Analysiert man anhand dieses Kriteriums die Voraussetzungen in den einzelnen Bundesländern (soweit sie durch die Teilnahme an der Befragung unverzerrt widergespiegelt wird), so ergibt sich folgendes Befundmuster (vgl. Grafik 4; eine Signifikanzprüfung hierzu erscheint nicht angemessen).

Die günstigsten Einschätzungen - mit jeweils über 90 \% flächendeckender Umsetzung - ergeben sich danach für teilnehmende Regionen aus dem Saarland, Schleswig-Holstein, Rheinland- Pfalz, Baden-Württemberg und Bayern, vergleichsweise ungünstige Umsetzungsdichte für Nieders- achsen (53\%) und Sachsen-Anhalt (62\%).

\section{Zusammenfassung}

Als ein wichtiges Gesamtergebnis ist zunächst festzuhalten, dass die Radfahrausbildung, die durch Verkehrserziehungsdienste der Polizei (zum Teil in Kooperation mit Schulen, s. u.) durchgeführt wird, derzeit eine nach wie vor sehr hohe Umsetzungsreichweite aufweist. Flächenübergreifend werden 85,7 \% aller Klassen im Rahmen des jeweiligen Zuständigkeitsbereichs der Dienststelle bis zum Abschluss der Grundschule komplett erreicht (wobei allerdings in mehreren Bundesländern diese Umsetzungsdichte, offensichtlich zum Teil sogar beträchtlich, unterschritten wird). Bezogen auf Mittelwertsangaben („\% aller Klassen erreicht") weist die Radfahrausbildung im Bundesgebiet - mit 93,6\% - eine nach wie vor sehr hohe Umsetzungsreichweite auf. Gegenüber

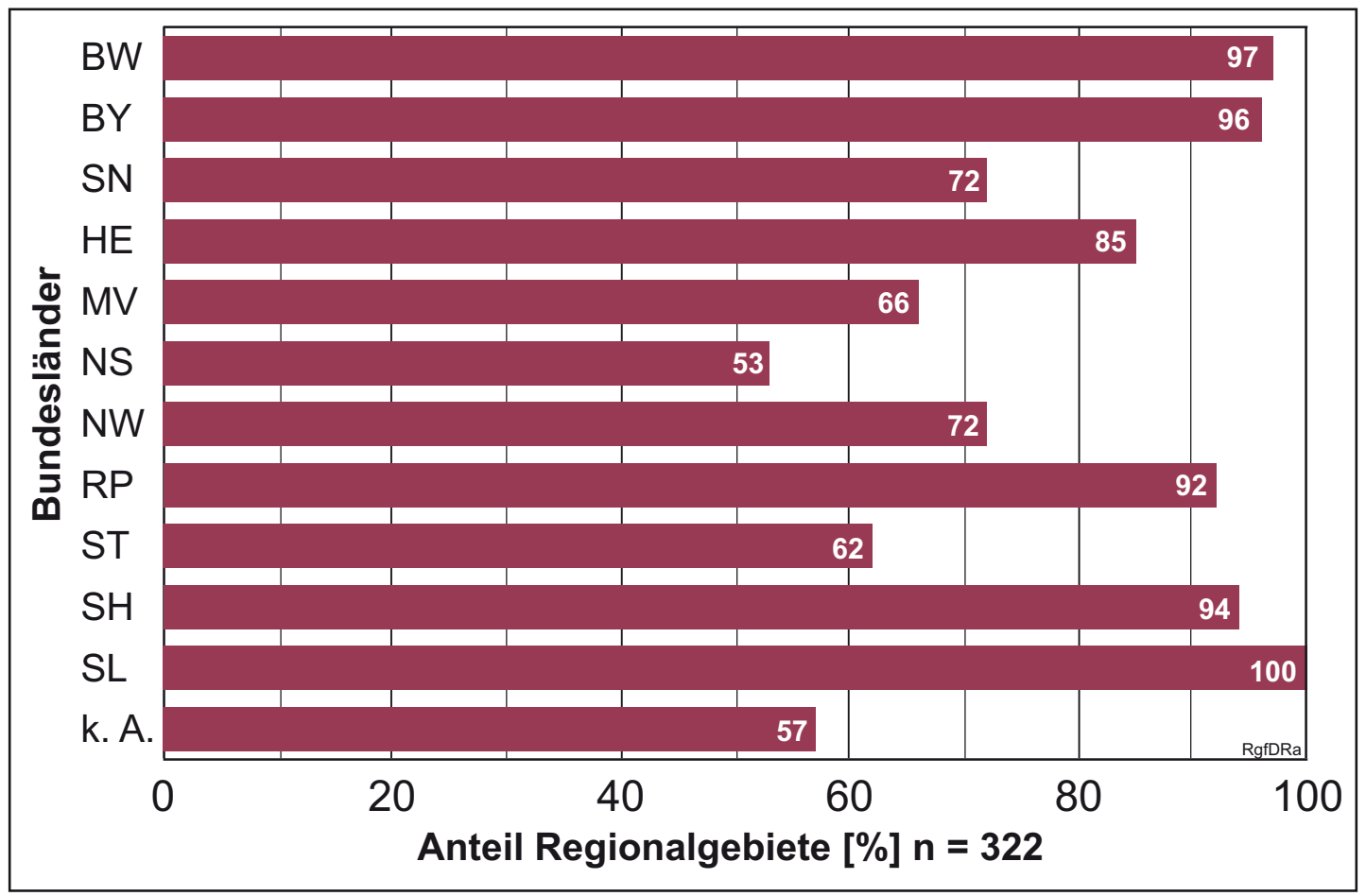

Grafik 4: Anteil der Regionalgebiete pro Bundesland mit flächendeckender Durchführung der Radfahrausbildung innerhalb des persönlichen Zuständigkeitsbereichs 


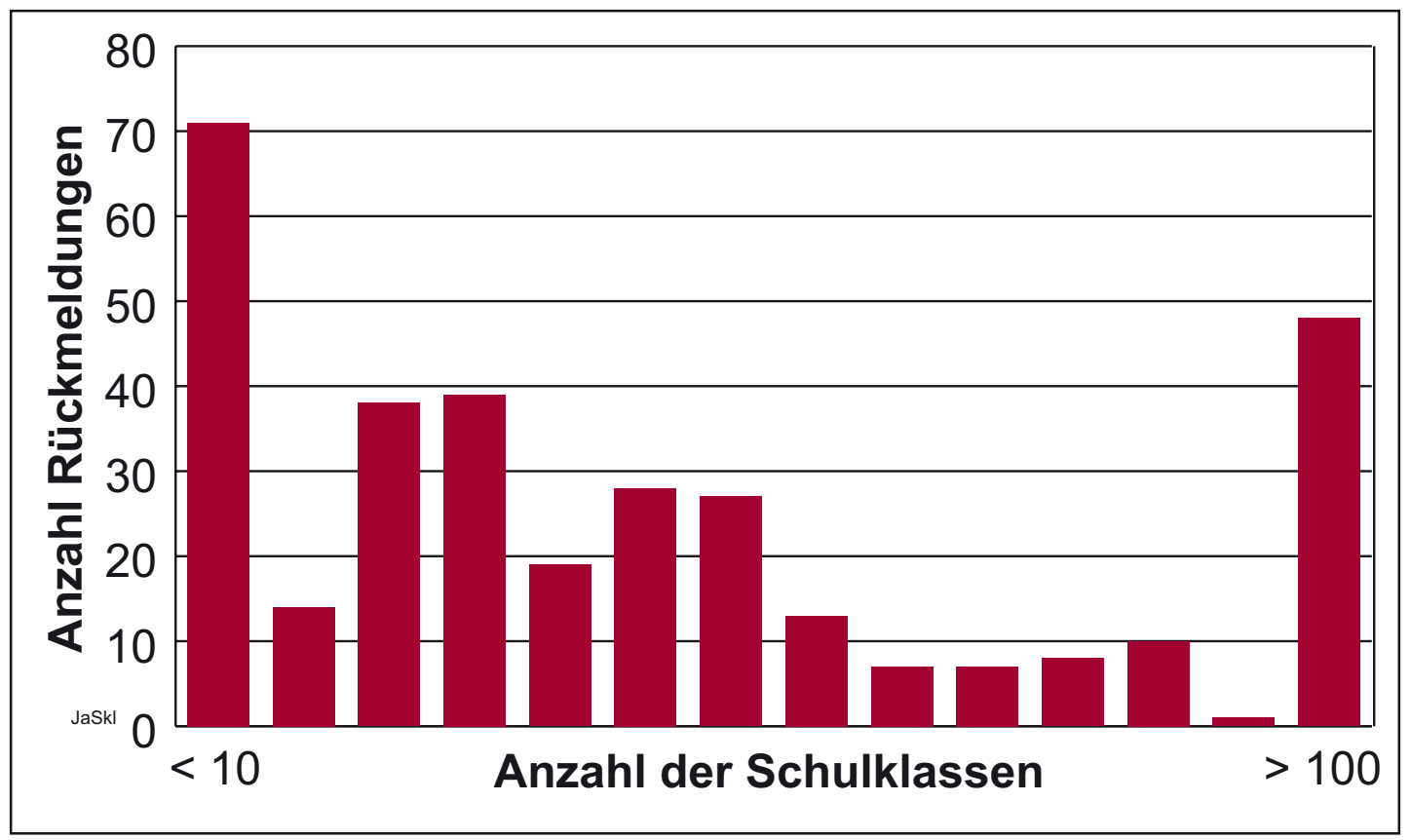

Grafik 5: Zahl der jährlich ausgebildeten Schulklassen

der Ersterhebung 1997 finden sich danach keine Verschiebungen (Ersterhebung: 93,5\%).

\section{Radfahrausbildung durch Schule oder Polizei}

Zunächst beachtenswert ist, dass die Gesamtzahl der im laufenden Jahr ausgebildeten Klassen im unteren Bereich einen auffälligen Häufigkeitsschwerpunkt aufweist (vgl. Grafik 5).

Eine wesentliche Ursache dafür ist, dass ein nicht ganz unerheblicher Anteil der Befragungen nicht durch Dienststellen der Polizei, sondern von Schulen beantwortet wurde; bei Inspektion der freien Kontaktinformationen (siehe 2.1) werden bei weniger als 10 jährlich durchgeführten Radfahrkursen die Fragebogen praktisch durchgehend von Lehrern bearbeitet (durchaus meist mit dem Hinweis einer Kooperation mit der Polizei). Legt man dieses Kriterium zugrunde, so ergibt sich als einigermaßen verlässlicher Schätzwert, dass 23 \% der erfassten Regionen die Radfahrausbildung (schwerpunktmäßig) schulintern organisieren, in der weit überwiegenden Mehr- zahl der Regionalgebiete - 76,7 \% - liegt die vorrangige Zuständigkeit bei Polizeiverkehrslehrern. Dabei zeigen sich quantitativ sehr beträchtliche Unterschiede zwischen den Bundesländern. Während in Baden-Württemberg, MecklenburgVorpommern, Saarland oder Sachsen ausschließlich, in Bayern, Hessen oder Sachsen-Anhalt die Rückläufe ganz überwiegend (jeweils mehr als $85 \%$ ) von Polizeidienststellen stammen, wurden am anderen Verteilungsende, in Schleswig- Holstein, gerade 8 \% der Rückläufe von Polizeistellen bearbeitet, andere flächenmäßig große Bundesländer wie Nordrhein-Westfalen, Niedersachsen oder Rheinland-Pfalz nehmen eine Mittelposition (mit schätzungsweise einem Drittel der Ausbildungen unter Zuständigkeit der Schulen) ein.

\section{Lernorte der Ausbildung und Prüfung}

Eine wichtige, in früheren Jahren (etwa bei einer vorausgehenden Fassung der DVWRadfahrausbildungskonzeption 1991) teilweise noch kontrovers diskutierte Frage bezieht sich auf die Einbeziehung von realen 


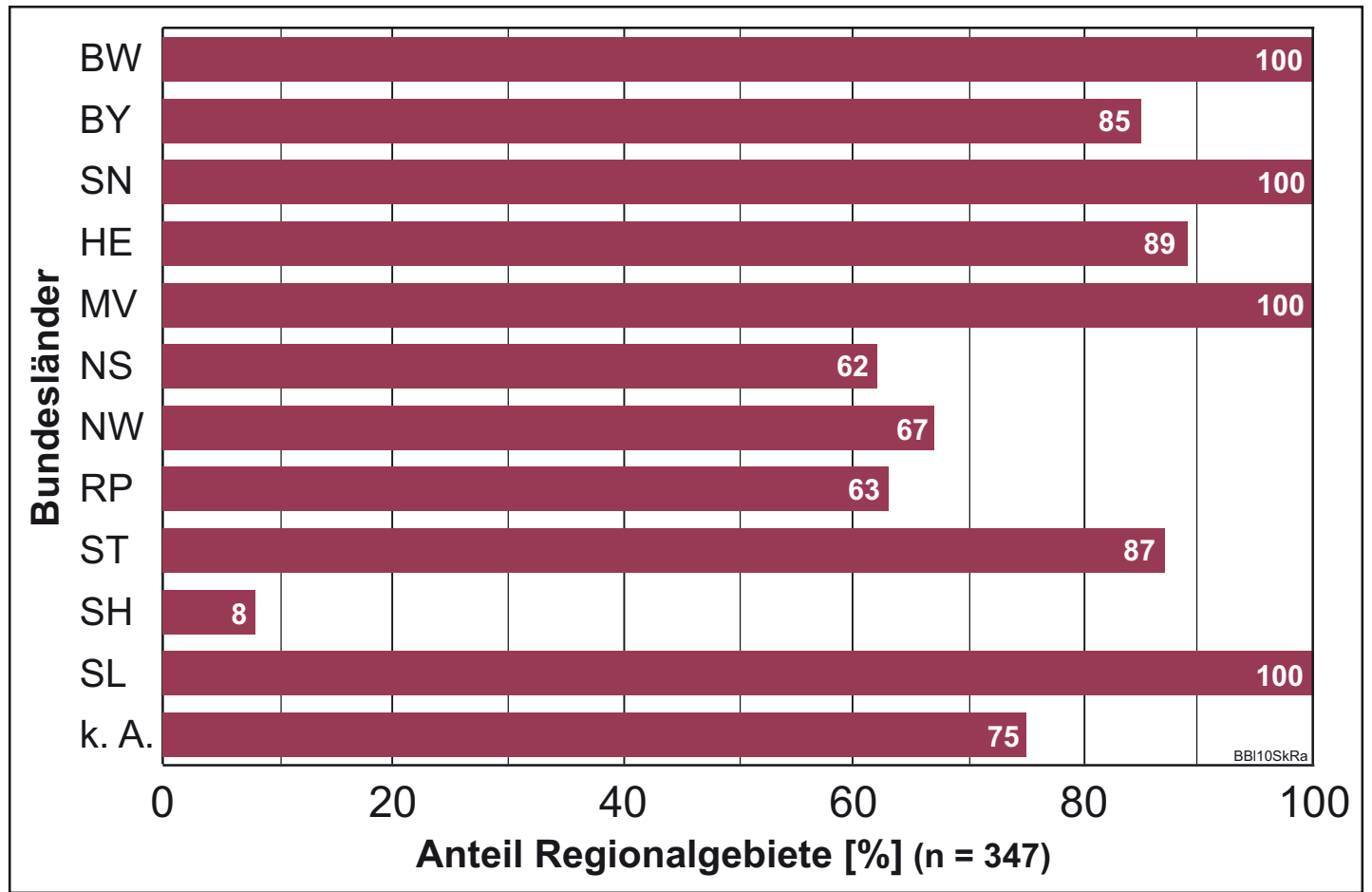

Grafik 6: Anteil der Befragten pro Bundesland, die in mindestens 10 Schulklassen im laufenden Schuljahr die Radfahrausbildung durchgeführt hatten

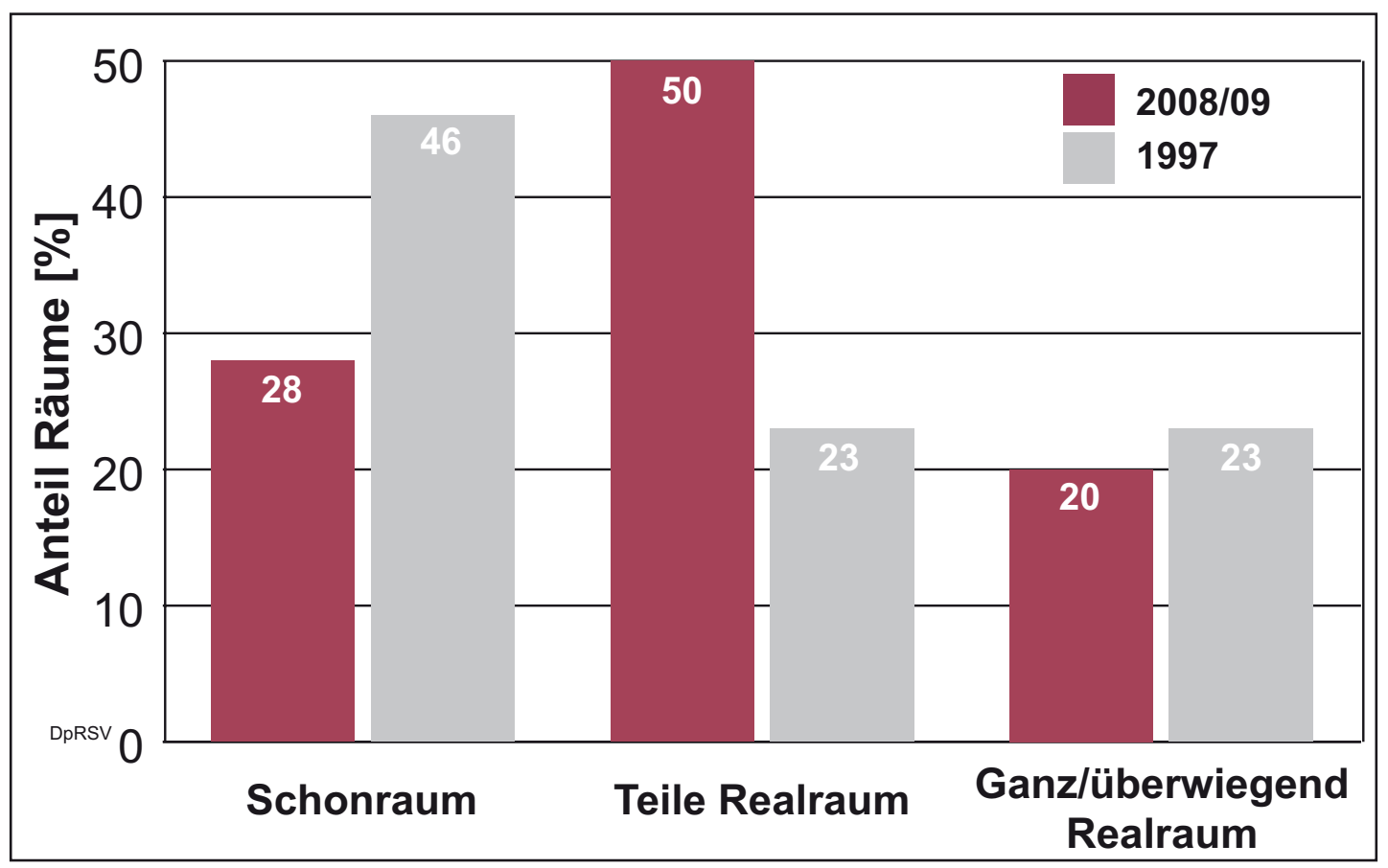

Grafik 7: Häufigkeitsverteilung für Durchführung der praktischen Radfahrausbildung und -prüfung in Schonräumen oder realen Verkehrsräumen (in \%). 


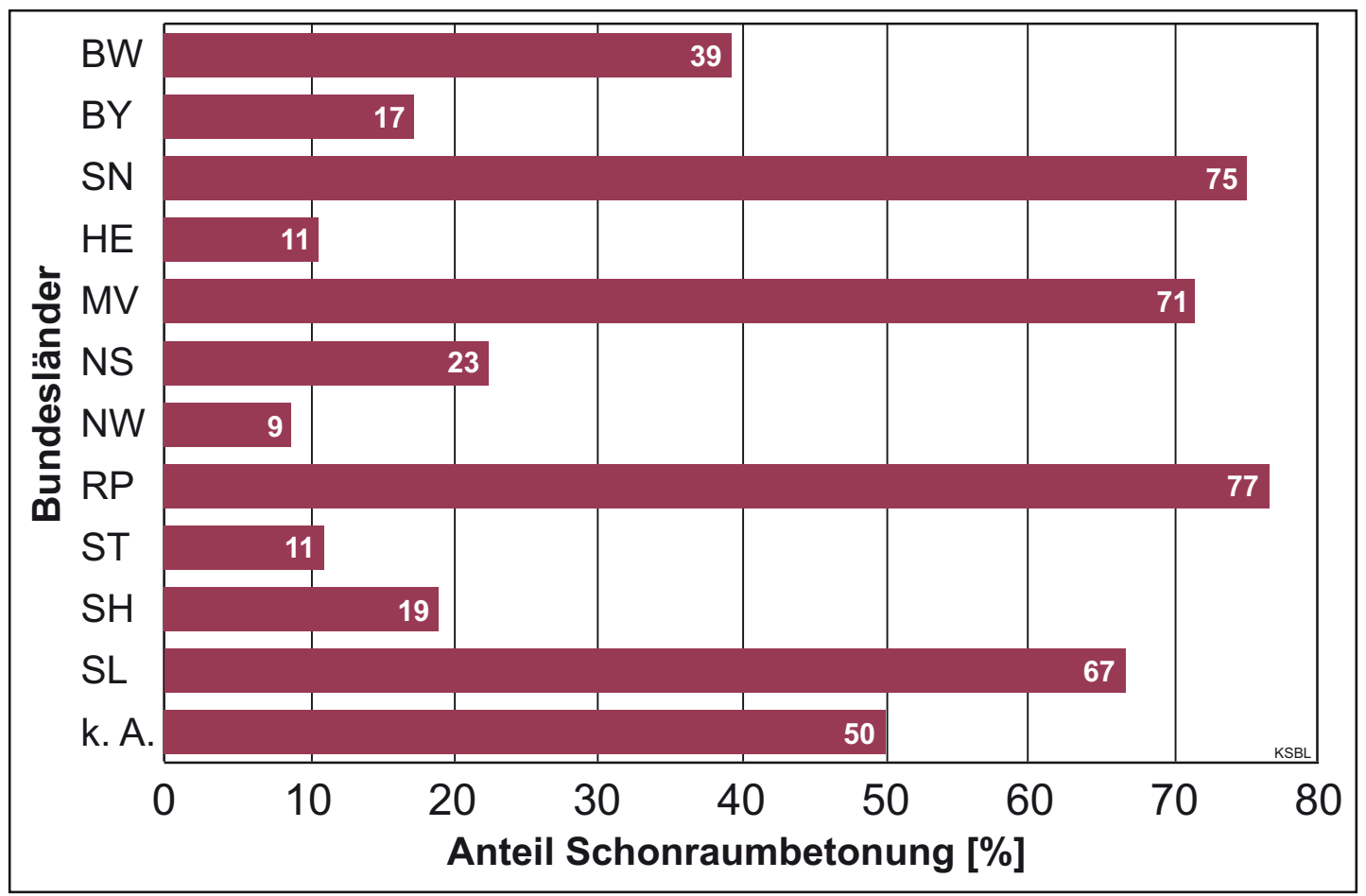

Grafik 8: Klare „Schonraum“-Betonung als Lernort (in \% der erfaßten Regionen).

Verkehrsräumen (in Abgrenzung zu „Schonräumen" der stationären oder mobilen Jugendverkehrsschulen, Schulhöfen) bei Gestaltung der fahrpraktischen Lernangebote. Dazu liefert die Befragung folgende Ergebnisse (vgl. Grafik 7).

Festzuhalten ist hier eine deutliche Veränderung gegenüber der Ersterhebung 1997:

Heute werden in $50 \%$ der Regionalgebiete die Radfahrausbildung und - prüfung überwiegend oder zumindest teilweise im realen Verkehrsraum durchgeführt (Ersterhebung: 23,1\%). Der Anteil der Ausbildungen, die überwiegend oder ausschließlich in stationären oder mobilen Jugendverkehrsschulen durchgeführt werden, ist von 46,2 \% (1997) auf 28,2 \% zurückgegangen. Eine schwerpunktmäßige oder ausschließliche Durchführung im Realraum findet sich in 20,2 \% der Umsetzungen, etwa gleich häufig wie vor 10 Jahren (23,1\%). Dabei findet sich keine klarere Beziehung zwischen dem Urbani- sierungsgrad der Region (groß-, kleinstädtisch, ländlich) und der Einbeziehung von realen Verkehrsgegebenheiten in die Ausbildung (Chi-Ou. (6) $=4,48$, n. s.).

Als regionale Extremposition fällt das Bundesland Schleswig-Holstein auf, in dem mit 91,3 \% ein Realraum-Durchführungsschwerpunkt berichtet wird, gefolgt von Hessen und Sachsen-Anhalt mit je $89 \%$.

Umgekehrt findet sich eine klare „Schonraum“Betonung als Lernort (vgl. Grafik 8) in Rheinland-Pfalz, Sachsen, Mecklenburg-Vorpommern und dem Saarland.

\section{Zusammenfassung}

(1) Gegenüber der Ersterhebung 1997 sind recht deutliche Veränderungen bezüglich der Lernorte festzustellen, wobei vor allem „gemischte“ Durchführungsabläufe 
(mit Durchführungsanteilen sowohl auf Schonflächen als auch im realen Verkehrsraum) erheblich zugenommen haben.

(2) Dennoch bestehen aber auch gegenwärtig noch sehr unterschiedliche Umsetzungsformen; Empfehlungen, bei denen eine bestimmte Form der ortsbezogenen Organisation der Radfahrausbildung und -prüfung als „Sollwert“ vorgegeben wird, dürften danach derzeit unverändert keine Aussicht auf generell ausreichende Akzeptanz finden.

\section{Allgemeinere Rahmenbedingungen der Radfahrausbildung}

Bei einer „gemischten“ Form der Durchführung wird in den offenen Antworten deutlich, dass dabei oft lediglich begrenztere Teile der Ausbildung, ausschließlich die Prüfungsfahrten oder Angebote sogar erst nach der Ausbildung im realen Verkehrsraum erfolgen.

Die qualitativen Hinweise über allgemeine Rahmenbedingungen der Radfahrausbildung beziehen sich vor allem auf die Frage:

„11. Sonstige Anmerkungen, Hinweise, z. B. Besonderheiten der Kursdurchführung bei Ihnen"

\section{Beachtenswerte Einzelnennungen}

- „Üben im Realverkehr wird mit einbezogen und angeboten! Aber selten genutzt!"

- „Realverkehr nach Prüfung“

- „Übung im Realverkehr wird bei großen Schwierigkeiten (der ganzen Klasse) in Absprache mit Lehrkraft abgesagt; eine weitere Übungseinheit erscheint bzw. ist zweckmäßiger!“

- „Übungen: 4 × 2 Doppelstunden im Schonraum (einschließlich Prüfung); 1 × 2 Doppelstunden im Realverkehr"
- „Buskinder machen keinen Realverkehr (Absprache mit SchA)“

- „gestiegene Durchfallquote nach Wegfall 4.E. Dadurch zu viel Inhalte und zu wenig Übungszeit. Besser: 4 ÜE - Prüfung - Realverkehr. Realverkehr sollte beibehalten werden“

- „Prüfung organisatorisch bedingt leider immer im Dezember, Prüfung ist nur bedingt durchführbar"

- „regelmäßig Gesprächskreis Schule/Berater Polizei“

- „vorbereitende Übungen in 3. Klasse durch Klassenlehrer"

- „Eltern sind eingeladen teilzunehmen, werden teilweise mit einbezogen"

- „zusätzliche Übungsstunden zu bestimmten Terminen an Samstagen, Behindertenausbildung wird dieses Jahr neu eingeführt."

\section{Sonstige Hinweise}

- „Kinder haben oft zu große Fahrräder, vor allem in der 4.Klasse"

- Kinder von Förderschulen sind lernfreudiger und disziplinierter. Ganztagsschulkinder haben weniger Auffälligkeiten."

- „die Kinder, oftmals Mädchen, beherrschen das Fahrrad noch nicht; zu wenig Übung, daher fehlt die Konzentration auf den anderen Fahrzeugverkehr und Gegebenheiten, Fahrräder zu groß und zum Teil nur mit Handbremsen ausgestattet."

- „teilweise ändert sich das Sozialverhalten der Kinder zum Negativen; kein Interesse mehr an der Radfahrausbildung gerade von solchen Kindern."

- zum Kurs sollten unabdingbare Übungen zur Wahrnehmungsschulung eingeplant werden."

\section{Zusatzangebote im Rahmen der Radfahraus- bildung}

Immerhin $52 \%$ der Befragten geben inhaltliche Hinweise zu der Frage: 
„7. Führen oder führten Sie sonstige Untersuchungen (z. B. Sehtests) mit Kindern in Zusammenhang mit der Radfahrausbildung durch? Ggf. auch: Warum jetzt nicht mehr?"

Die Antwortquote ist deutlich höher als in der Erstbefragung (damals $18 \%$ der Befragten).

Qualitative Angaben beziehen sich vorrangig auf Sehtests (im Altersbereich zwischen Ende der Grundschulzeit und Übergang auf weiterführende Schulen bildet sich in Zusammenhang mit dem Körperwachstum insbesondere Myopie heraus, die zunächst von Kindern und Eltern oft nicht frühzeitig erkannt wird; ferner bleiben auch weitere, oft von Geburt an bestehende Beeinträchtigungen der Sehfunktionen, etwa Varianten der Farbenblindheit, bisweilen unentdeckt).

Die Aussagen lassen sich schwerpunktmäßig in folgende Gruppen einteilen:

\section{Zustimmung a) durch Eltern}

z. B.: „Sehtest durch Eltern“

z. B.: „Die Eltern nehmen die Voruntersuchungen sehr ernst und bringen ihre Kinder, falls sie ohnehin in Behandlung sind, regelmäßig zum Augenarzt. Für die Schule besteht kein Handlungsbedarf."

\section{Zustimmung b) durch Optiker}

z. B.: „teilweise durch Klassenlehrer (Sehtest durch Optiker)“

z. B.: "Sehtest mit Optiker in Schule“

z. B.: „Seit 2003 wird bei jeder Klasse nach der Prüfung der Sehtest durch einen Optiker durchgeführt."

\section{Nein}

z. B.: „Nein (x-fache Nennungen)“

z. B.: „keine medizinische Untersuchung“

z. B.: „Nein, in Hamburg nicht vorgesehen“

z. B.: „zurzeit nicht vorgesehen, wäre aber sinnvoll" z. B.: „nein, weil kein Optiker sich zu kostenlosem Sehrtest bereit erklären lässt“

z. B.: „nein; nach Rücksprache mit den jeweiligen Schulen - kein Bedarf"

z. B.: „In Rheinland-Pfalz gibt es keine Sehtests!"

\section{liegt in der Zuständigkeit der Schulen}

z. B.: „Lehrkräfte werden auf Sehtest hingewiesen, diese führen die Sehtests durch."

z. B.: „Nein. Sehtests liegen im Aufgabenbereich der Schulen und somit der Klassenleitung."

z. B.: „Polizei nicht, aber die Schulen ja (Sehtest und Reaktionstest)“

\section{zeitlich nicht realisierbar}

z. B.: „Für Sonderprogramme fehlt es der JVS an Zeit!!“

z. B.: „Zeitlich nicht machbar. Hinweise an Eltern bei Übergabe der Ausweise"

\subsection{Motorische Schwächen von Kindern}

\section{Gesamteinschätzung}

Ein wichtiger Teil der Befragung bezog sich auf Einschätzungen über die Häufigkeit auftretender motorischer Schwächen von Kindern innerhalb der Schulklassen, die die Radfahrausbildung durchlaufen, beginnend mit einer Trendeinschätzung:

„3. Meine Einschätzung zum Trend in den zurückliegenden Jahren: Die Zahl von Kindern, die bei der Radfahrausbildung durch mangelhafte Körperbeherrschung/Radfahrfertigkeiten auffallen (hat erheblich abgenommen ... hat erheblich zugenommen)."

Die Häufigkeitsveränderung ,in den zurückliegenden Jahren" liefert dabei folgendes Urteilsmuster durch die befragten Verkehrserzieher (vgl. Grafik 9). 


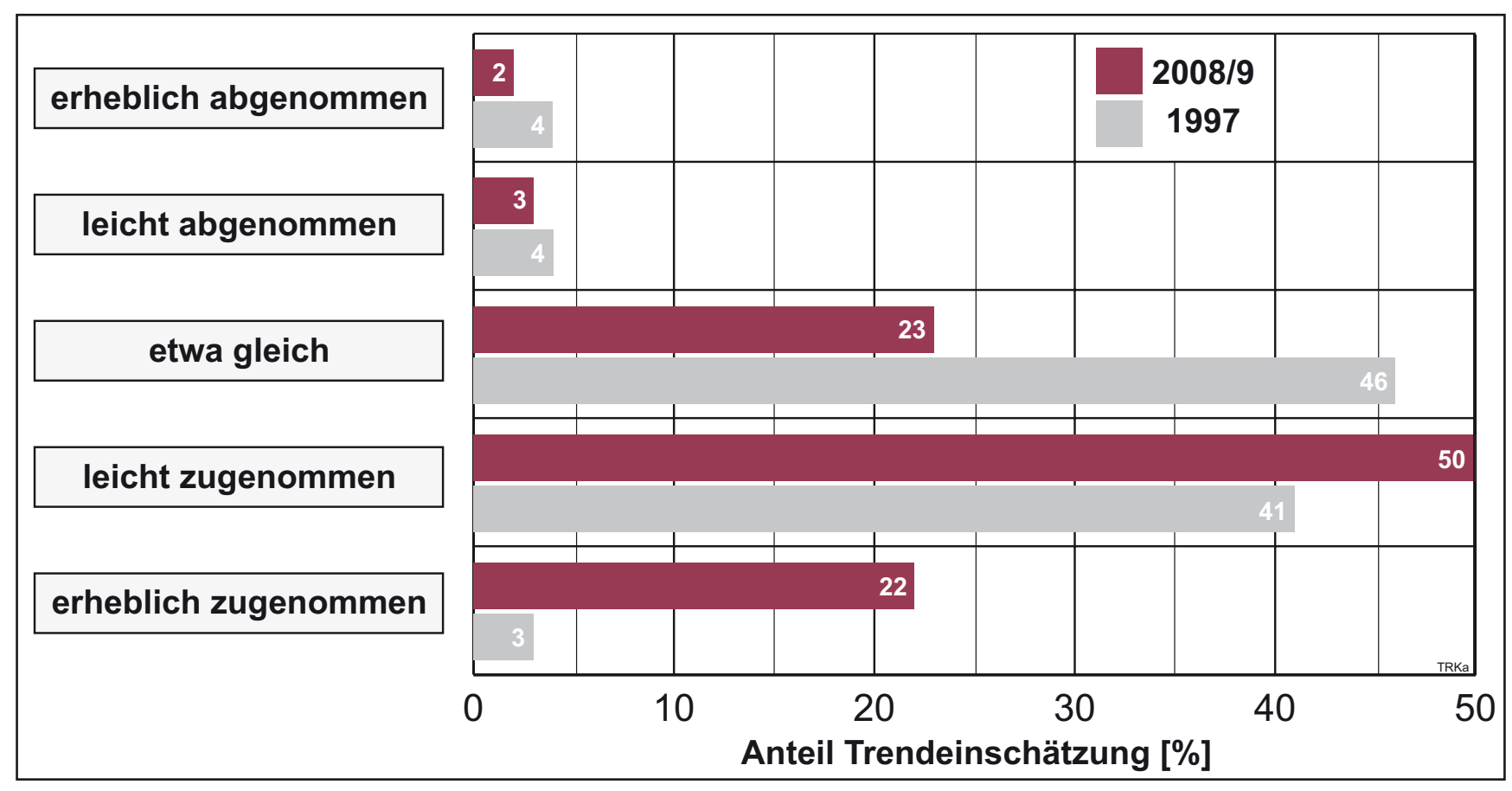

Grafik 9: Trendeinschätzung zur Zahl von Kindern, die bei der Radfahrausbildung durch mangelhafte Körperbeherrschung/Radfahrfertigkeit in den zurückliegenden Jahren auffielen. Mangelhafte Körperbeherrschung / Radfahrfertigkeiten hat: erheblich abgenommen, leicht abgenommen ...

Zunächst ist festzuhalten, dass nur mehr rund ein Fünftel (23\%) (Ersterhebung: knapp die Hälfte (46,1 \%) der befragten Verkehrserzieher den Eindruck haben, dass die Anzahl von Kindern, die bei der Radfahrausbildung durch motorische Schwierigkeiten auffallen, etwa gleich geblieben sei.

Innerhalb der Teilgruppe der Befragten, die zahlenmäßige Veränderungen beobachtet haben, zeigt sich darüber hinaus aber ein sehr prägnantes, hoch zeitstabiles Befundmuster: Einem Anteil von 8,3 \% (Ersterhebung: 5 \%) der Befragten, die davon ausgehen, dass in der zurückliegenden Zeit ein Problemrückgang festzustellen war, stehen 72 \% (1997: 45,6 \%) gegenüber, die einen ansteigenden Trend beobachten; der vergleichsweise geringe Anteil von Nennungen, die auf eine „erhebliche“ Zunahme verweisen (3,9\%), spricht wiederum für eine quantitativ nicht sehr ausgeprägte
Prävalenzänderung. Auch der große Anteil von Nennungen, die auf eine „erhebliche“ Zunahme verweisen (22\%, rund ein Fünftel der Fälle, 1997 nur $3 \%)$, spricht für eine quantitativ stark angestiegene Prävalenzänderung.

Die Asymmetrie im Anteil zu- bzw. abnehmender Einschätzungen ist statistisch selbstverständlich hoch gesichert ( $p<0,1 \%)$.

In einer zusätzlichen Frage wurden Einschätzungen über den Anteil von Schulklassen erbeten, in denen (a) „keines der Kinder deutliche Geschicklichkeits- und Fertigkeitsbeeinträchtigungen aufweist“, (b) „,ünf oder mehr Kinder innerhalb der Klasse" deutliche Beeinträchtigungen aufweisen.

Im Gesamtdurchschnitt werden für knapp die Hälfte (43 \%, 1997 waren es 49 \%) der Klassen keine motorisch auffallenden Problemkinder 

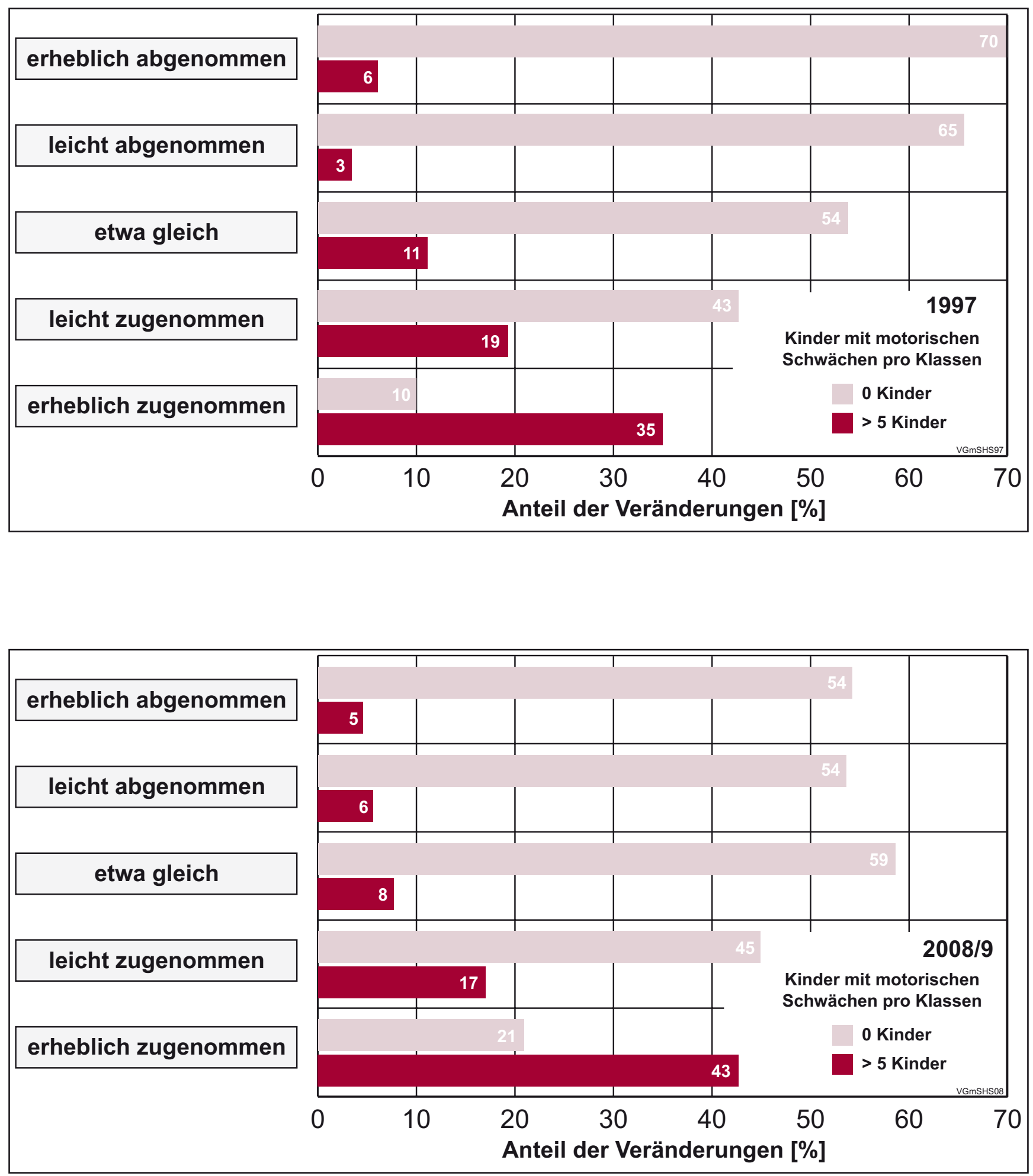

Grafik 10:Einschätzung über Veränderungen im Gesamttrend von motorischen Schwächen und Schätzung von Häufigkeitsanteilen innerhalb von Schulklassen, Differenzierung nach Einschätzung des Gesamttrends (Fr. 3) 


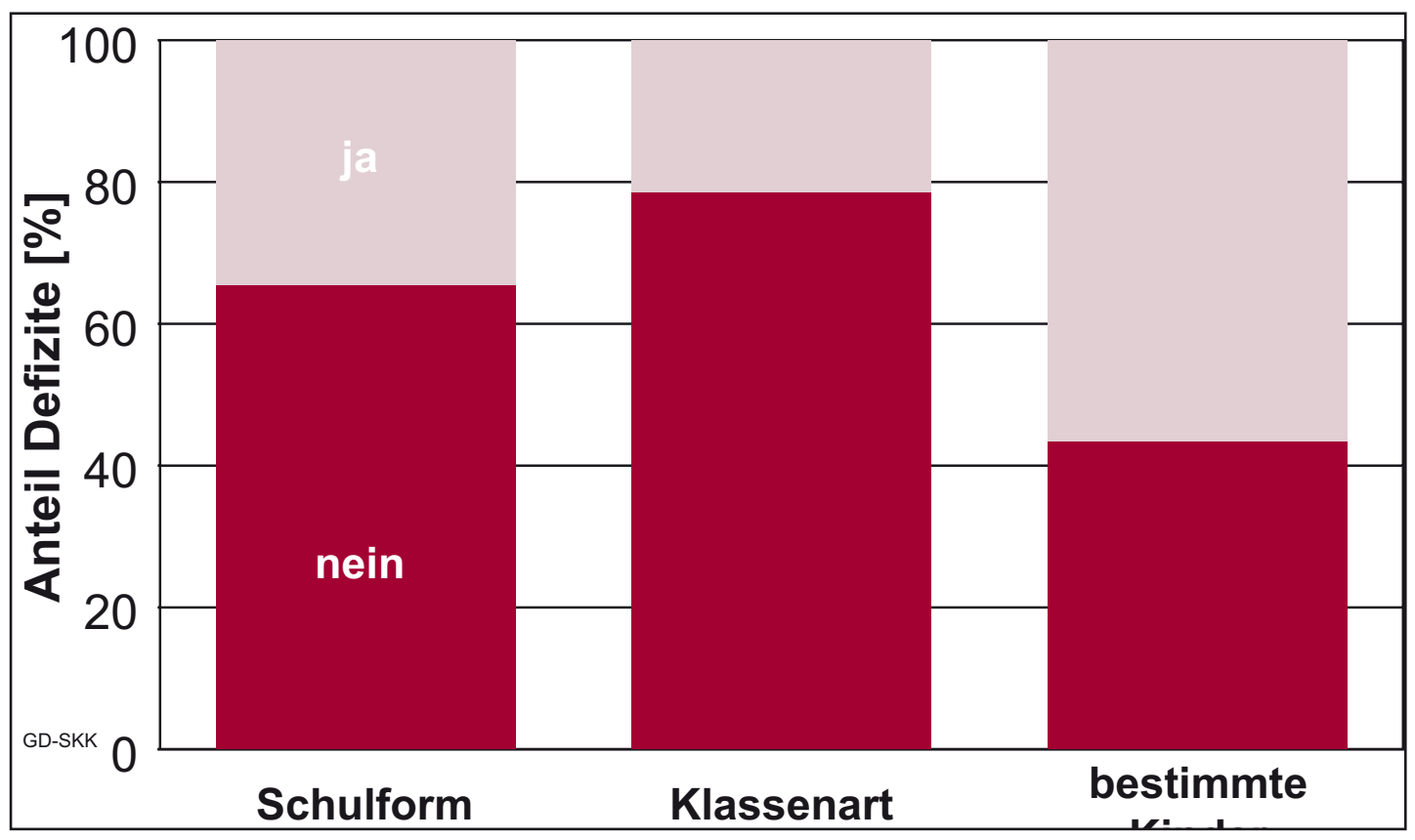

Grafik 11: Haben Sie den Eindruck, dass Geschicklichkeitsdefizite unterschiedlich häufig auftreten: In bestimmten Grundschuleinrichtungen (ohne Sonderschulen) / In bestimmten Schulklassen (ohne Förderklassen) / bei bestimmten Kindern? (Fr. 9)

beobachtet. Umgekehrt finden sich nach der Erfahrungen der polizeilichen Verkehrserzieher quantitativ nicht selten (mit einen Mittelwert von $20 \%$ ) Schulklassen (im Vergleich dazu 14 \% 1997), bei denen eine größere Anzahl von mindestens fünf Kindern deutliche Beeinträchtigungen aufweisen. In beiden Fällen auch deutet das auf einen Anstieg der motorischen Auffälligkeiten der Schüler hin.

Vergleicht man die Befundlage 2008/09 mit 1997 nach einer differenzierteren Aufschlüsselung (vgl. Grafik 10), so ergibt sich indes ein klar verändertes Gesamtbild:

Während vor 10 Jahren sich die Einschätzung einer klaren Verbesserung vs. Verschlimmerung der Schwächen vorrangig daran orientierte, ob im aktuellen Beurteilungszeitraum motorisch „problemfreie“ Klassen anzutreffen waren, so hat derzeit dieses positive Bewertungskriterium mittlerweile erheblich an Gewicht einge- büßt (vgl. die verminderte Spreizung der Werte in der 1. Datenspalte 2008/09).

Zusammenfassend ist also festzustellen, dass die Befunde bezüglich der Verbreitung von motorischen Schwächen der Kinder im Vergleich zum Zeitpunkt der Ersterhebung eine erhebliche, weitere Verdichtung der Problemsituation zum Ausdruck bringen.

\section{Hinweise auf motorische Problemverdichtung}

Ein recht beträchtlicher Anteil der Befragten gibt auch inhaltliche Hinweise, inwieweit in bestimmten Grundschuleinrichtungen, Schulklassen oder bei bestimmten Gruppen von Kindern Geschicklichkeitsdefizite vergleichsweise häufiger auftreten (Frage 9 (Anhang); vgl. Grafik 11).

Entsprechende Hinweise werden weniger für Grundschuleinrichtungen und Schulklassen also vielmehr bei bestimmten Gruppen 
von Kindern gesehen; die Antwortverteilung unterscheidet sich dabei nur wenig von den Einschätzungen der Ersterhebung (Einrichtungen: 35 \% inhaltliche Hinweise, Schulklassen $22 \%$, bestimmte Gruppen von Kindern $57 \%)$.

\section{Qualitative Einschätzungen}

Die qualitativen Einschätzungen liefern ein recht differenziertes Erklärungsbild (allerdings ist die Abgrenzung zwischen den drei Teilaspekten oft unscharf):

\section{(a) Schuleinrichtungen}

Bei möglichen Erklärungen warum Geschicklichkeitsdefizite in bestimmten Schulen unterschiedlich häufig auftreten, wurden schwerpunktmäßig genannt:

\section{- Lage Wohnort/Lage Schule}

z. B.: „Wohnortbedingungen, die Radfahren // nicht// ermöglichen"

z. B.: „topografische Lage der Schule. Qualität sportunterricht"

z. B.: „Mangelnde Möglichkeit der Nutzung des Rades durch Gefälle/Steigung/starken Verkehr."

\section{- Stadt/Land}

z. B.: „Meiner Beobachtung nach sind Kinder aus ländlichen Bereichen geschickter als Stadtkinder."

z. B.: „Land viel, viel besser.“

z. B.: „Stadt/Land-Gefälle“ (x-fache Nennungen)

z. B.: „An Stadtschulen sind mehr Kinder, die nicht so gut fahren können."

z. B.: „im ländlichen Bereich sind Schüler sicherer, da mehr Bewegung und Spiele draußen."

z. B.: „Stadtkinder. Landkinder fahren offenbar noch weit mehr mit dem Rad“

\section{- hoher Ausländeranteil}

z. B.: „Hoher Ausländeranteil, vor allem Mädchen“ z. B.: „Innenstadtschulen mit überwiegendem Ausländeranteil“

z. B.: „Migrantenfamilien, Anteil arabisch-/ türkischstämmiger Familien, asiatische Familien"

\section{darüber hinaus}

\section{- Mangel an Bewegung}

z. B.: „Mangel an Bewegung, sitzen vor Computer und Fernseher"

z. B.: „mangelnde Bewegung, Übung“

z. B.: „wenig Bewegung der Kinder in der Freizeit" - geringe Förderung durch die Eltern

z. B.: „Eltern fördern die Kinder nicht ausreichend (milieuabhängig)“

z. B.: „Soziale + Wohlstandsvernachlässigung“

\section{- (sozial)schwache Familien}

z. B.: „finanzschwache Familien“

z. B.: „Soziales Umfeld“

z. B.: „Soziale Schichten“

\section{(b) Bestimmte Schulklassen}

Bei möglichen Erklärungen warum Geschicklichkeitsdefizite in bestimmten Schulklassen unterschiedlich häufig auftreten, wurden schwerpunkt mäßig genannt:

\section{- sozial schwache Familien/Ausländer}

z. B.: „Klassen mit hohem Ausländeranteil“

z. B.: „Soziale Schwerpunkte“

z. B.: „finanzielle Probleme“

z. B.: „bei erhöhtem Anteil ausländischer Mitbürger"

\section{- geografische Lage}

z. B.: „geografische Lage, Ausländeranteil“

z. B.: „Innenstadtlage, sehr hügelige Wohnumgebung"

z. B.: „Differenzen bei sogenannten (Land- und Stadtkinder), Kinder „vom Land“ fahren geschickter"

z. B.: „Kinder, die in verkehrsreichen Gebieten leben" 


\section{- mangelnde Unterstützung durch die El-} tern/Lehrer

z. B.: „mangelnde Unterstützung der Eltern und Lehrer"

z. B.: „soziale Strukturen; einige Kinder werden durch Eltern nicht genug gefördert!“

z. B.: „Engagement von Eltern, teilweise Lehrern"

\section{- mangelnde Vorbereitung durch die Lehr- kräfte oder im Kindergarten}

z. B.: „Vorbereitung durch die Lehrkräfte“

z. B.: „Vorbereitung in jüngeren Jahrgangsstufen wurde an Schulen nicht durchgeführt/bzw. von Lehrern nicht durchgeführt"

z. B.: „Mangelndes Interesse der Lehrer“

z. B.: „geringe Motivation der/des Sachkundelehrerin/lehrers"

[z.B.: „zu wenig Praxis in den Vorschuljahren"]

\section{(c) Bestimmte Kinder}

Bei möglichen Erklärungen, warum Geschicklichkeitsdefizite bei bestimmten Kindern unterschiedlich häufig auftreten, wurden überwiegend genannt:

\section{- Mädchen mit Migrationshintergrund}

z. B.: „Mädchen mit Migrationshintergrund“ (x-fache Nennungen)

z. B.: „ausländischen Mädchen“ (x-fache Nennungen)

z. B.: „Mädchen kurdischer Abstammung“

\section{- Migranten}

z. B.: „Migrationshintergrund, soziale Probleme"

z. B.: „Migrationshintergrund“ ( $x$-fache Nennungen)

z.B.: „Jungen mit Migrationshintergrund überdurchschnittlich gut; Mädchen mit Migrationshintergrund - unterdurchschnittlich" z. B.: „Kinder mit muslimischem Glauben (Islam)"

- sozial schwache Familien, Kinder die kein Fahrrad besitzen

z. B.: „aus sozial schwachen Familien, Migranten"

z. B.: „Ausländerkindern - Migranten/Asylanten, sozial schwächere Familien“

z. B.: „sozial Benachteiligte (besitzen kein eigenes Fahrrad), Kinder bildungsferner Elternhäuser, bei denen keine Heranführung an das Radfahren durch die Eltern erfolgt"

z. B.: „Kinder ohne Fahrrad“

z. B.: „Kinder aus sozial schwachen Familien haben oft kein Fahrrad daheim!"

z. B.: „Kinder aus schwachen sozialen Verhältnissen, die z. B. gar kein Rad besitzen“

\section{darüber hinaus wurden nennenswert ge-} nannt

- überbehütete Kinder, Bewegungsmangel

z. B.: „überbehütet, Fernseh-/Computer-Kinder“

z. B.: „Kinder, die immer mit dem Auto zur Schule kommen"

z. B. „kein Üben mit Eltern; Überbehütung aus Angst vor Unfällen; Bewegungsmängel“

z. B.: „Kinder die allzu lange durch Eltern behütet werden"

z. B.: „überbehütete Kinder; werden stets mit Pkw zur Schule gebracht“

\section{- übergewichtige Kinder}

z. B.: „übergewichtige Kinder“

z. B.: „Übergewicht, mangelndes Körpergefühl“

z. B.: „, Kinder mit starkem Übergewicht“

\section{Interessante Einzelaussage}

„Es stellt sich heraus, dass oftmals Kinder die nicht schwimmen können auch nicht

Radfahren können." 

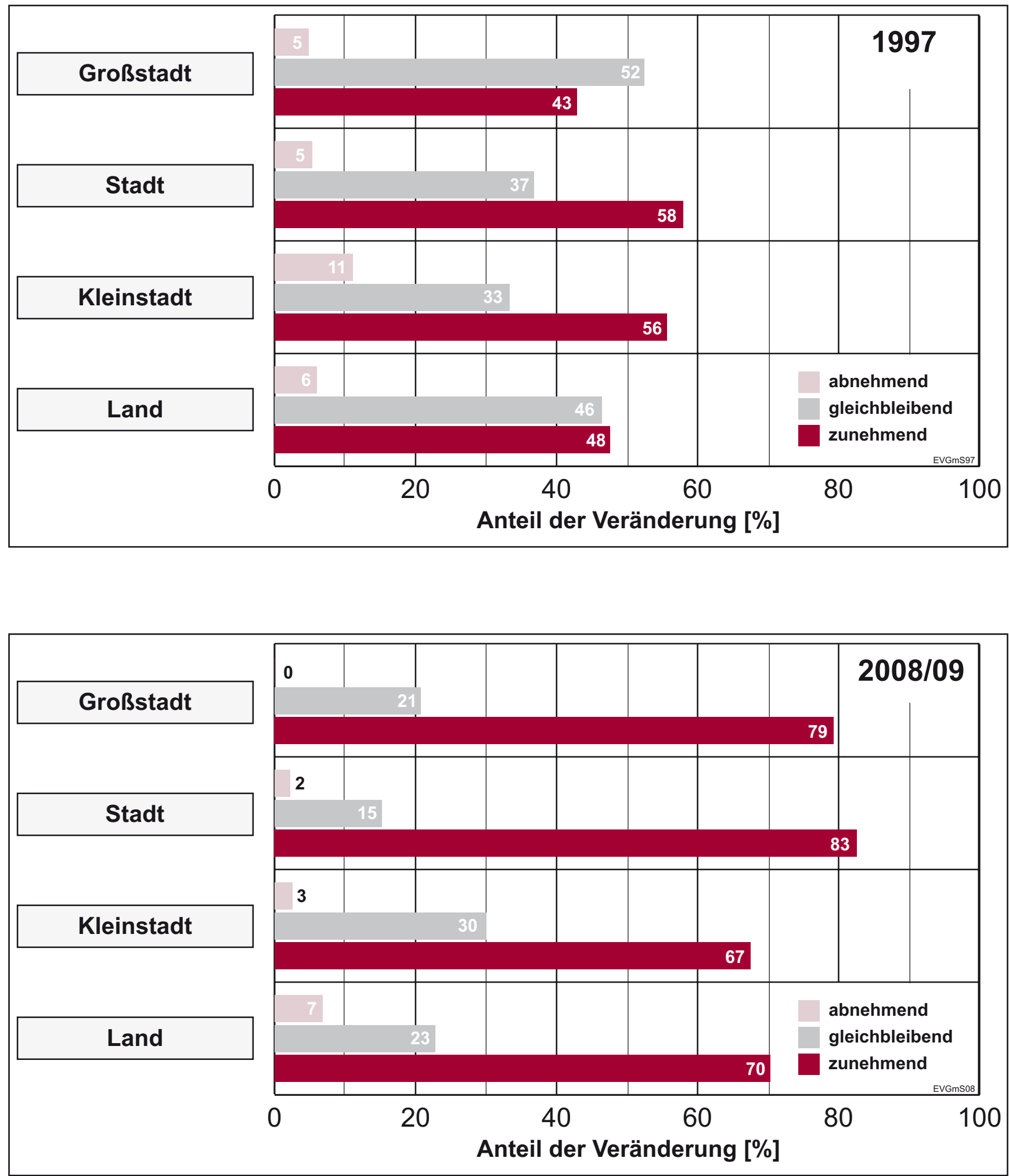

Grafik 12: Einschätzung über Veränderungen im Gesamttrend von motorischen Schwächen, Differenzierung nach Urbanisierungsgrad der Region ( $n=133$; in \%) 


\section{Unterschiede zwischen städtischen und eher ländlichen Regionalgebieten}

Insgesamt ist nach der Befundlage davon auszugehen, das der Umfang bestehender psychomotorischer Schwächen, die sich insbesondere auch auf die Voraussetzungen zur selbständigen Verkehrsteilnahme beim Radfahren auswirken, für Kinder aus Großstadtgebieten und aus städtischen Kerngebieten am weitesten fortgeschritten ist.

Die Befundlage zu wahrgenommenen Problemveränderungen findet sich in Grafik 12.

Danach zeigt die Befundlage deutlich: Trendveränderungen zeigen sich für alle Regionaltypen, derzeit vergleichsweise am ausgeprägtesten bei Kindern, die in Mittelstädten leben.

\subsection{Stellenwert von motorischen Schwierigkeiten für die Anforderungsbewältigung in der Radfahrausbildung}

Ein weiterer Teil der Befragung sollte Hinweise darauf liefern, welcher Stellenwert vorhandenen motorischen Unsicherheiten von Kindern für die fahrpraktische Ausbildung zukommt. Dazu sollte zunächst frei beantwortet werden:

„4. Wenn motorische Schwächen bei bestimmten Kindern auftauchen: Bei welchen Ausbildungsteilen/Fahraufgaben der Ausbildung führt dies besonders häufig/deutlich zu Schwierigkeiten im Verhalten? (Bitte kurz erläutern).“

Qualitative Angaben machten hierzu fast alle Befragten (97\%).

Die inhaltlichen Aussagen verweisen dazu schwerpunktmäßig auf folgende Probleme, die den motorisch schwächeren Kindern besondere Schwierigkeiten bereiten:

- Abbiegen (v. a. das Einhändigfahren und das Umschauen und dabei die Spur halten dabei) z. B.: „,beim Abbiegen“ ( $x$-fache Nennungen)

z. B.: „Beim Linksabbiegen - Umschauen während der Fahrt lässt manche Kinder in den "Gegenverkehr" fahren!"

\section{- Anfahren und Bremsen}

z. B.: „Bremsen wurde nicht gelernt"

z. B.: „bremsen, anfahren, aufsteigen“ ( $x$-fache Nennungen)

\section{- Auf- und Absteigen}

z. B.: „aufsteigen von rechts“

\section{- Gleichgewicht halten}

z. B.: „Anfahren - Gleichgewicht halten; Bremsen - sicheres Bremsen und Stand"

\section{- Koordinationsschwierigkeiten, Schwierig-} keiten bei Mehrfachanforderungen

z. B.: „mangelnde Koordination; Kinder sind vielfach nicht in der Lage Handzeichen zu geben (nicht in der Lage einhändig zu fahren); Kinder sind nicht in der Lage sich umzusehen, verziehen das Fahrrad nach links"

z. B.: „grundsätzlich bei Übungen mit Handzeichen, bei komplexen Verkehrssituationen, grundsätzlich bei Mehrfachhandlungen (Linksabbiegen, freies Fahren)" (x-fache Nennungen)

\section{- generelles Spurhalten}

z. B.: „Fahrradbeherrschung und Spurhalten, Handzeichen geben, Linksabbiegen!"

Seltener wurden ferner erwähnt

- teilweise allgemeine Mängel bei der Fahrradbeherrschung, damit verbunden Konzentrationsschwierigkeiten auf die Anforderungen 
z. B.: „Fast in jeder Klasse gibt es 1 - 2 Kinder, die noch gar kein Fahrrad fahren können; große Schwierigkeiten gibt es beim Einhändigfahren (Handzeichen)“

\section{- Fettleibigkeit/Übergewicht}

z. B.: „mangelnde motorische Fähigkeiten, teilweise wegen Übergewicht“

\section{- Probleme bei bestimmten Übungen, wie Achterfahren etc.}

z. B.: „Kette, Achter beim Jugend Fahrrad Turnier"

z. B.: „Slalom, langsames Fahren“

z. B.: „Fahrten im Geschicklichkeitsparcour“

z. B.: „Spurbrett, Schrägbrett“

Einige „deutliche“ Beispiele für Auswirkungen, die den Befragten besonders deutlich in Erinnerung geblieben sind (Frage 5 (Anlage); qualitative Angaben machen $42 \%$ der Befragten):

\section{- Kinder die nicht (richtig) Radfahren kön- nen und deshalb teilweise vom Unterricht ausgeschlossen werden bzw. Kurs leidet unter der notwendigen Verzögerung durch Rücksichtnahme}

z. B.: „Bei erheblichen Mängeln werden Kinder aus der Schulung genommen (zum Schutz der anderen)."

z. B.: „Es kommt durchaus mal (selten) vor, dass Schüler(innen) vom Kurs ausgeschlossen werden, weil sie einfach nicht gelernt haben Fahrrad zu fahren"

z. B.: „Einzelne Kinder müssten erst das Radfahren erlernen"

z. B.: „Kind musste bei leichter Steigung bereits absteigen und schieben und fiel beim Absteigen auf die Fahrbahn. War bei dem Kurs Hindernis für die anderen Teilnehmer, die sich auch mit „lahme Ente" u. ä. beschwerten. Als das Kind dann zum Schluss fuhr, musste der Kurs lange warten."

\section{- es muss mit manchen Kindern, die starke} Mängel aufweisen, intensiver geübt werden (meist zu Lasten der anderen Teilnehmer)

z. B.: „Bei Aussicht auf erfolgreiche Behebung der Mängel erfolgt intensive Beschäftigung mit diesem Kind. Dies geht zu Lasten der anderen Kursteilnehmer."

z. B.: „wenn Einschränkung zu groß dann Einfachübungen mit Lehrer/in" (x-fache Nennungen)

\section{- Linksabbiegen und dabei Handzeichen geben/Einhändigfahren}

z. B.: „Kind war nicht fähig einhändig zu fahren“

z. B.: „Das Kind gibt beim Linksabbiegen Handzeichen und verliert das Gleichgewicht"

\section{- Gleichgewicht halten}

z. B.: „Gleichgewicht halten beim einhändigen Fahren. Gleichzeitiges Umsehen ist dann unmöglich"

\section{- Gefährdung von sich und anderen durch unsicheres Fahren}

z. B.: „Kind fuhr in Böschung, weil nicht handhabungssicher auf abschüssiger Straße"

z. B.: „Kind musste aus Sicherheitsgründen von Schulung und Prüfung ausgeschlossen werden.

Es lässt sich danach sagen, dass sehr grundsätzliche Anforderungen, die beim Radfahren laufend in Alltagssituationen sicher beherrscht werden müssen, bei Kindern mit motorischen Fertigkeitsschwächen nicht zuverlässig bewältigt werden können; dies betrifft generell die Fähigkeit der sicheren Spureinhaltung bei seitlicher oder rückwärtsgewandter Orientierung, vor allem in Kombination mit beabsichtigten Richtungsänderungen und deren Anzeige (auch nach rechts). Auswirkungen von praktischen Fertigkeiten der Radfahrtechnik betreffen demnach nicht seltene Sondersituationen, in denen ein besonders hohes Überforderungsrisiko besteht, sondern vielmehr Grundqualifikationen für die Bewältigung von ständig auftretenden Teilaufgaben bei 
der Verkehrsteilnahme. Diese Gesamtbewertung stimmt weitgehend mit den Einschätzungen aus der Ersterhebung von 1997 überein.

Zusätzlich ist anzumerken, dass ausgesprochene Problemsituationen zwar im theoretischen Unterrichtsteil (oder bei nicht beabsichtigtem Eintritt während der Praxisphasen) besprochen, ohnehin meist nicht gezielt eingeübt werden können (nach den curricularen Voraussetzungen, den üblicherweise verfügbaren zeitlichen Ressourcen, aber auch aus praktischen - etwa versicherungsrechtlichen - Gründen); dazu zählt beispielsweise unvermutetes, regelwidriges Verhalten von Fußgängern, die ohne Orientierung einen Radweg betreten, oder von Kraftfahrern, etwa regelwidriges "Schneiden“ von Radfahrern beim Rechtsabbiegen, bilden typische Problemsituationen, die im Erleben von geübten Radfahrern als häufiger eintretende Bedrohung gefürchtet werden (vgl. [3]).

\subsection{Organisatorische Maßnahmen zur Sicherung der Kursdurchführung}

Ein weiterer Teil der Befragung dient der Abklärung, inwieweit die Teilnahme von Kindern, die Schwierigkeiten bei der Radbeherrschung haben, die Kursdurchführung erschwert, und inwieweit polizeiliche Verkehrslehrer durch speziellere organisatorische Maßnahmen sich auf diese Problemgruppe einstellen müssen. Zunächst sollte eingeschätzt werden:

"2. Was sind Ihre allgemeinen Erfahrungen: Für die Durchführung der Radfahrausbildung ergeben sich durch mangelhafte Körperbeherrschung und verminderte motorische Fertigkeiten beim Radfahren von bestimmten Kindern (keine Probleme .... regelmäßig erhebliche Probleme)."

Der Umfang der Problemeinschätzungen ergibt sich aus Grafik 13.

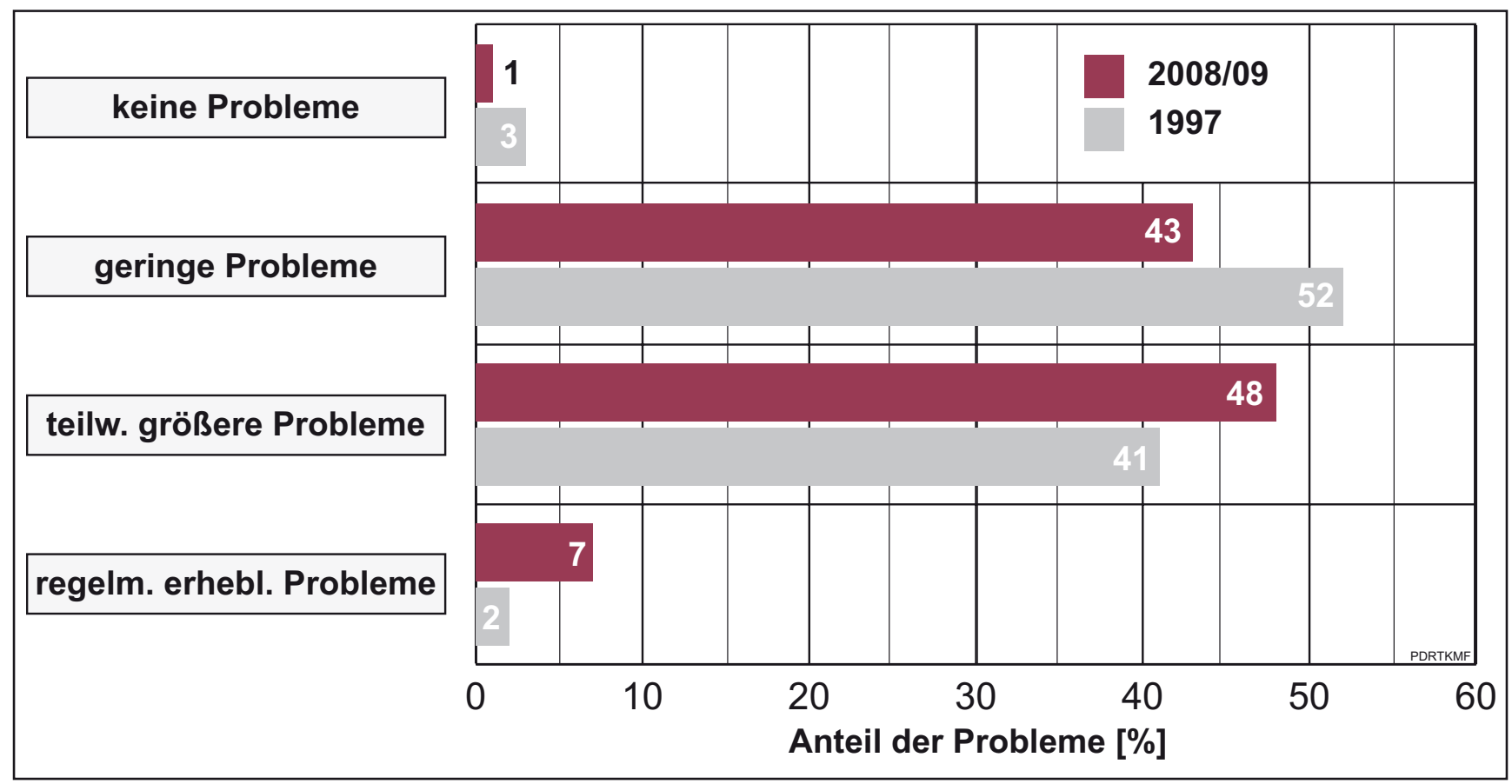

Grafik 13: Umfang von Problemen, die sich für die Durchführung der Radfahrausbildung durch Teilnahme von Kindern mit unzureichenden motorischen Fertigkeiten ergeben 
Danach zeigt sich, dass mehr als die Hälfte der Organisationseinheiten, die die Radfahrausbildung durchführen (55\%), größere oder sehr erhebliche organisatorische Probleme in diesem Zusammenhang sieht. Die Zunahme gegenüber der Ersterhebung (48\%) ist allerdings quantitativ nicht dramatisch.

Erwartungsgemäß wird dabei der Problemumfang weitaus kritischer eingeschätzt in Regionalgebieten, in denen in der letzten Zeit ein Anstieg bei der Anzahl von Kindern mit entsprechenden Fertigkeitsbegrenzungen beobachtet wird (Korrelationskoeffizient $r=0,47$ ).

„Erhebliche“ praktischen Schwierigkeiten bei der Ausbildung, die auf motorische Schwächen von Kindern zurückgeführt werden, finden sich in stärker städtisch geprägten Regionen etwas häufiger (Chi-Qu. (9) = 18,1, p<5\%). Hierzu in Einklang stehen qualitative Einschätzungen der Befragten (siehe 3.2).

Organisatorische Maßnahmen, um sich auf das Problem der motorisch schwächeren Kinder in der Radfahrausbildung einzustellen, berichten etwas mehr als $60 \%$ der Befragten. Die Antwortverteilung zu der Frage:

„8. Führen motorische Defizite von Kindern zu so erheblichen Schwierigkeiten bei der Durchführung von fahrpraktischen Ausbildungskursen, dass Sie sich durch organisatorische Maßnahmen darauf einstellen müssen? (nein, bisher nicht/kaum......ja, fast bei jedem Kurs)“

findet sich in der Grafik 15.

Immerhin fast $16 \%$ der Kurse erfordern nach den Einschätzungen der Befragten mithin häufig oder fast durchgehend spezielle organisatorische Maßnahmen, die sich aus den Problemen der Fahrfertigkeit von Kindern ergeben.
Die Maßnahmedichte ist dabei sehr eng mit der beschriebenen Problemhäufigkeit bei den Kindern (Frage 2) verknüpft ( $r=0,59$, s. s.), nicht so deutlich mit dem Umfang der geschätzten Veränderung der Problemdichte (Frage 3, $r=0,39$, s. s.).

Immerhin fast zwei Drittel der Befragten machen auch freie Angaben über diese Maßnahmen. Dabei zeigen sich folgende Hauptnennungen:

\section{- Einzelbetreuung/besondere Betreuung durch Lehrkraft/Kollegen/Eltern}

z. B.: „Einbindung der Lehrerschaft in praktisches Ausbildungsprogramm (Übung mit nicht radfahrenden Kindern)“

z., B.:,Einzelausbildung durch Lehrpersonal auf dem Schulgelände"

z. B.: „Durch den verstärkten Einsatz von Betreuern + Eltern“

z. B.: „größere Anzahl von Eltern, die Posten im Realraum beziehen, um die Kinder zu unterstützen"

z. B.: „Besondere Betreuung durch Lehrkraft; Fahren üben abseits des Übungsplatzes“

\section{- zusätzliche Übungen (z. B. auf dem Schul- hof etc.)}

z. B.: „Eltern werden gebeten, bis zum Tag der Lernzielkontrolle noch intensiv mit den Kindern zu üben"

z. B.: „Klassenlehrer übt mit dem Kind das Fahrrad fahren im Schonraum“

z. B.: „Nachmittags auf dem Schulhof ggf. 1 bis 2mal Einzeltraining; nachmittags ggf. Geschicklichkeits- $u$. Festigungstraining der motorischen Fähigkeiten in Schulprojekten"

\section{- Elternbenachrichtigung}

z. B.: „Einzelgespräch und Erklären von Fahrübungen, die das Kind zuhause machen kann. Evtl. Kontaktaufnahme zu den Eltern“ 


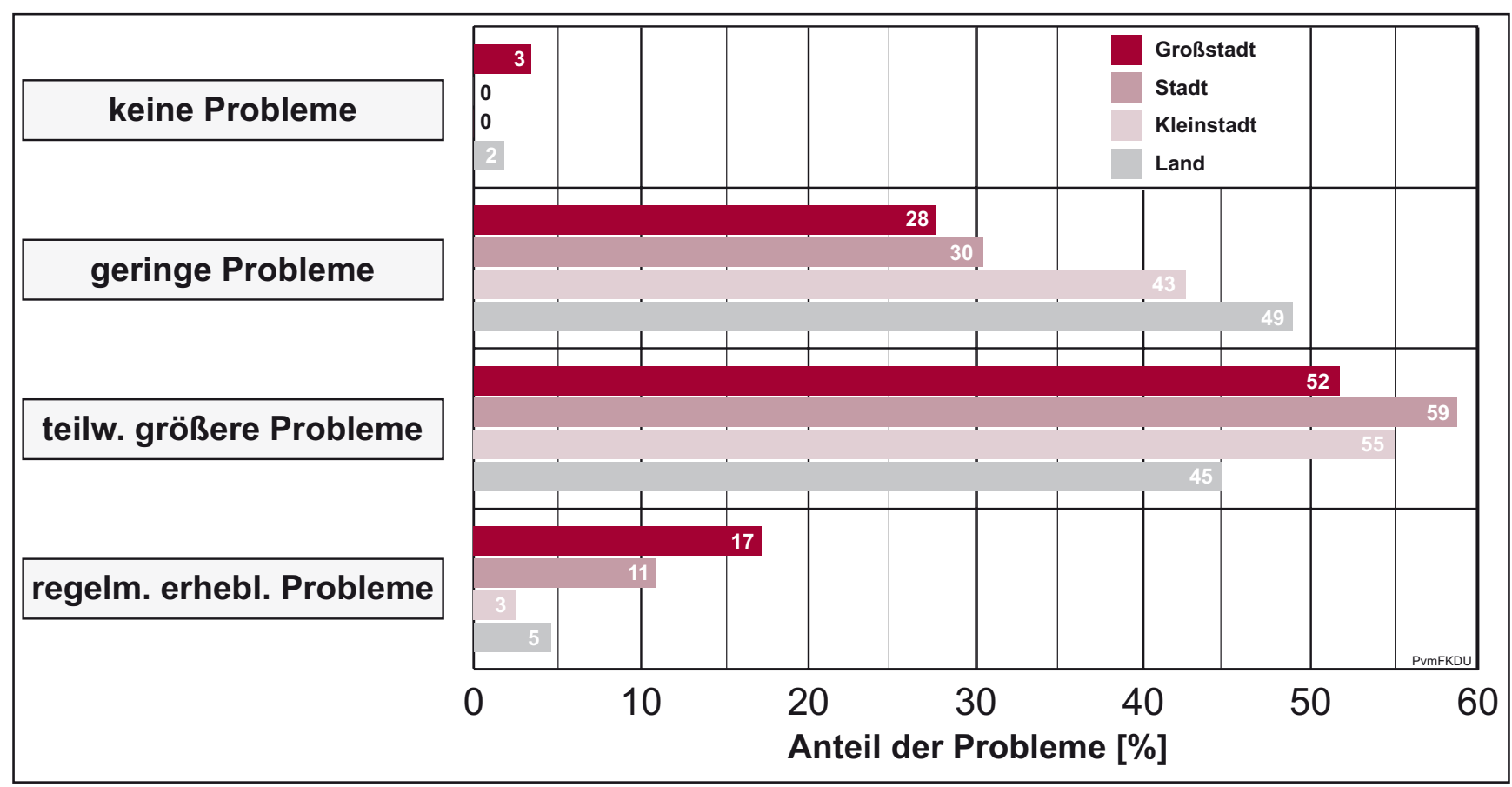

Grafik 14: Probleme wegen verminderter motorischer Fertigkeiten einzelner Kinder,Differenzierung nach Urbanisierungsgrad der Region (keine Vergleichstabelle für 1997 vorhanden)

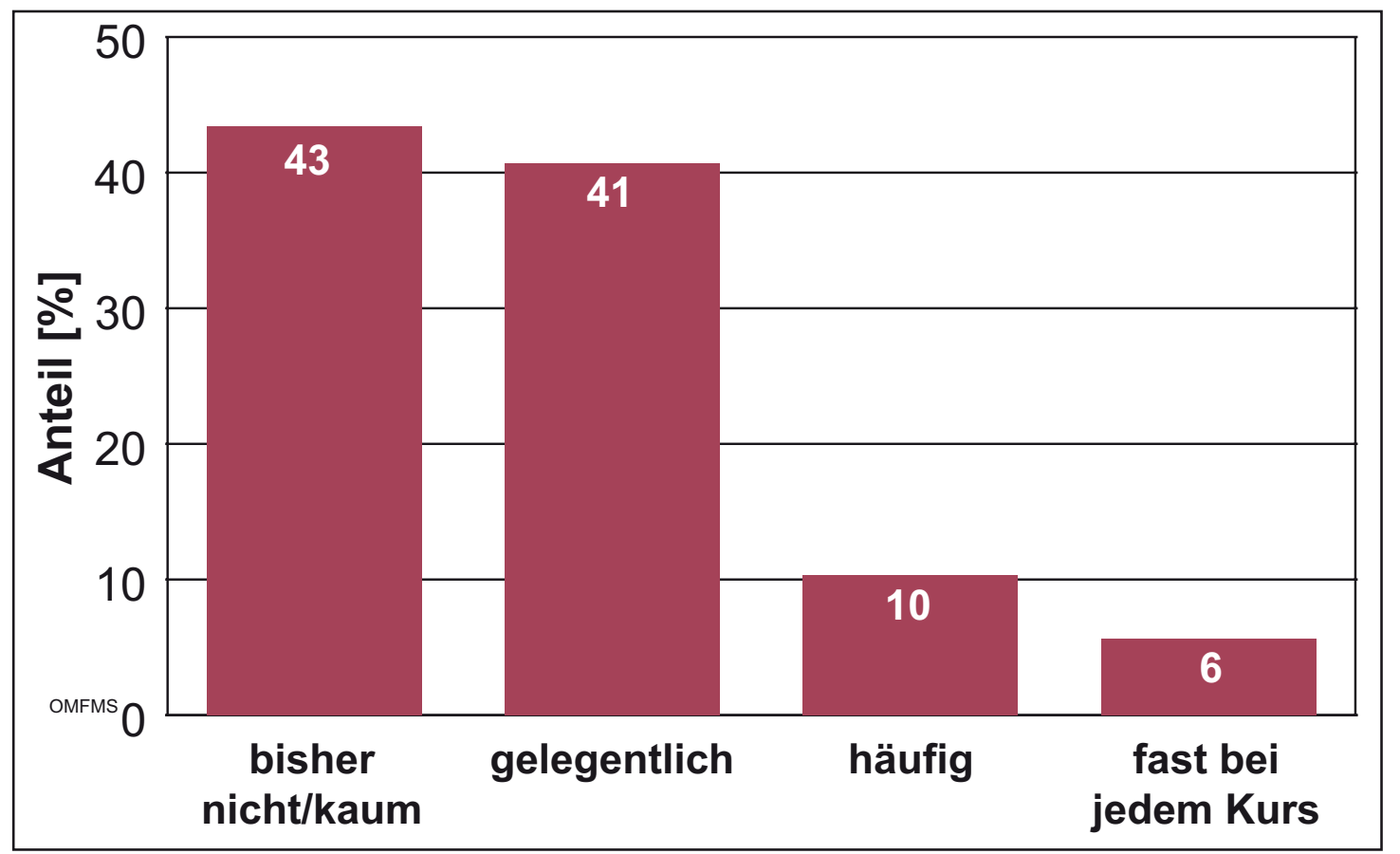

Grafik 15:Häufigkeitsverteilung erforderlicher organisatorischer Maßnahmen infolge fahrpraktischer motorischer Schwierigkeiten (keine Vergleichstabelle für 1997 vorhanden) 
z. B.: „Mitteilung an die Eltern, dass ihre Kinder motorische Mängel haben und die Eltern eigenverantwortlich damit umgehen sollten"

z. B.: „Eltern werden über Mängel der Kinder informiert - selbstverantwortlich!"

z. B.: „Mitteilung an die Eltern, welche Schwächen erkannt wurden"

z. B.: „vermehrtes Üben auf Schulhof. Hinweise an Eltern z. B. angemessene RadgröBe“

\section{darüber hinaus}

\section{- Ausschluss vom Realverkehr}

z. B.: „zusätzliche Übungseinheiten im Schonraum, kein Realverkehr"

z. B.: „Fahrten in Verkehrswirklichkeit 9. +10. Stunde nach der Prüfung entfallen"

z. B.: „keine Ausfahrt im Realverkehr"

z. B.: „keine Teilnahme an Ausbildungsteilen im Realverkehr"

\section{- Laufräder/Dreiräder/Roller}

z. B.: „Es wurde z. B. ein Roller (kein Cityroller) angeschafft. Kinder mit Gleichgewichtsstörungen konnten so anfangs Übungen leichter bewältigen“

z. B.: „Wir haben bei einem Rad die Pedale abgebaut, so dass es jetzt ein Laufrad ist"

z. B.: „Über einen Erwachsenenroller werden die Defizite beim Gleichgewicht schneller erlernt und geübt. Das Kind kann trotzdem an der Ausbildung teilnehmen, bekommt Mut und versucht früher oder später doch Fahrrad zu fahren."

z. B.: „Fahren mit Stützrädern, Roller“

z. B.: „Dreirad!“
Um mögliche organisatorische Ansatzpunkte genauer zu erfassen, wurden sechs Teilmaßnahmen abgefragt (vgl. Grafik 16).

Mit deutlichem Abstand am häufigsten werden danach derzeit Geschicklichkeitsprüfungen zur Radfahrbeherrschung durchgeführt, entweder unmittelbar bei Kursbeginn oder zeitlich früher (regelmäßig in 40 \% der einbezogenen Regionalgebiete). Hier zeigt sich keine Veränderung gegenüber der Ersterhebung 1997.

Im Vergleich zur Ersterhebung 1997 ist jeweils um fast die Hälfte zurückgegangen:

- die Zahl der Kurse, in denen regelmäßiger eine Einzelförderung während des Kurses erfolgt (18\%; 1997: $32 \%$; vgl. auch hierauf bezogene qualitative Angaben, s. o.) und

- die Zahl der regelmäßig angebotenen Nachkurse (17\%; 1997: 33 \%).

Die Interpretation dieser Veränderungen mag auch durch allgemeine Rahmenbedingungen der Radfahrausbildung und der Kursdurchführungen zu interpretieren sein (Frage 11, siehe 3.1).*

\section{Zusammenfassung}

Derzeit wird auf auftretende motorische schwächen der Kinder mit organisatorisch deutlich abweichenden und insgesamt erheblich reduzierten Maßnahmen reagiert, als dies noch vor 10 Jahren der Fall war. Vermindert ist vor allem die Zahl der Kurse, in denen regelmäßiger auch eine Einzelförderungen während des Kurses erfolgt (18\%; 1997: 32 \%) sowie das Angebot an Nachkursen (17\%; 1997: $33 \%)$. 

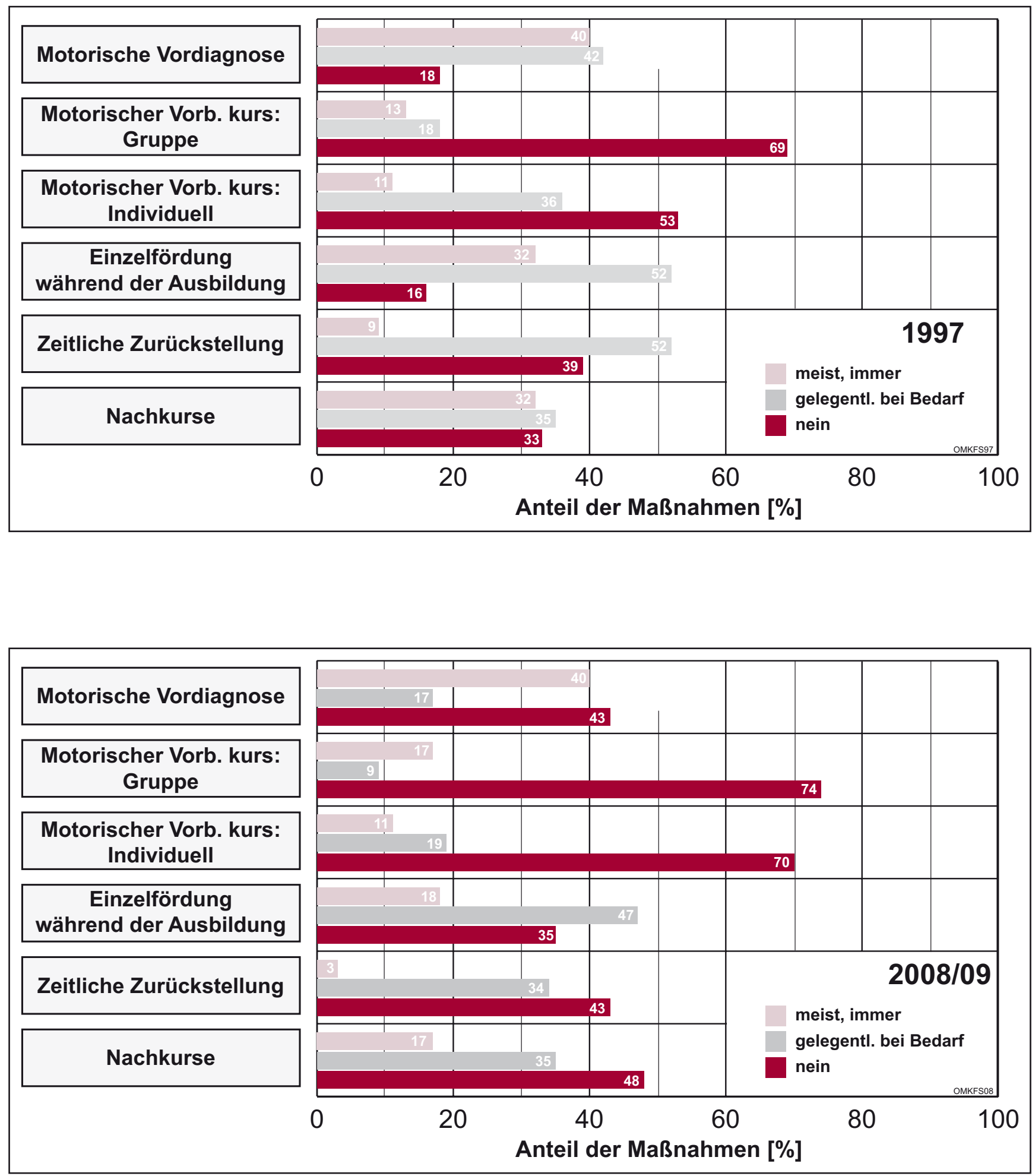

Grafik 16: Häufigkeitsverteilung durchgeführter organisatorischer Maßnahmen bei teilnehmenden Kindern mit erheblichen fahrpraktischen und motorischen Schwierigkeiten (in \%) 


\section{Diskussion der Hauptergebnisse und Zusammenfassung}

Diese Studie befasst sich mit zwei Fragebereichen:

(1) Durch eine bundesweite Befragung praxisnah tätiger Fachkräfte sollen Hinweise über den bestehenden Problemumfang der motorischen Schwächen von Kindern und deren Auswirkungen auf die Radfahrausbildung und über organisatorische Maßnahmen zur Sicherung der Kursdurchführung gewonnen werden.

Dass keine Anhaltspunkte über die Validität der durch Expertenbefragung gewonnenen Einschätzungen zur Verfügung stehen, sollte beachtet werden (zur wahrgenommenen Zunahme motorischer Schwierigkeiten sei etwa verwiesen auf den seit der Antike immer wieder geäuBerten "Topos", dass die Erziehung der Kinder heute schwieriger geworden sei im Vergleich zu „früher“; vgl. z. B. [5]).

(2) Da andererseits die aktuell durchgeführte Befragung eine inhaltlich weitgehende Wiederholung einer bereits vor etwa 10 Jahren im Auftrag der Deutschen Verkehrswacht durchgeführten Befragung [4] bildet, können zu den heute erhobenen Einzelthemen empirisch gestützte Einschätzungen über Umfang und Geschwindigkeit von Veränderungsprozessen abgeleitet werden und damit zugleich eine grobe Vorschau auf die zukünftige Problementwicklung.

\subsection{Stichprobenmerkmale}

\section{Stichprobe und Rücklaufquote}

Grundlage der Studie bildet eine schriftliche Anfrage bei $n=1.008$ VerkehrserziehungsDienststellen (Polizei, Schulen) aufgrund einer Adress-Datei der Deutschen Verkehrswacht mit weiter Streuung über das gesamte Bundesgebiet. Der Fragebogenversand erfolgte an der Jahreswende 2008/09. Die Rücklaufquote lag bei 36,5\% und war damit deutlich niedriger als die 1997 erreichte, sehr hohe Rücklaufquote $(74,9 \%)$. Für die Datenanalyse stehen $n=347$ vollständig bearbeitete Fragebogen zur Verfügung (Ersterhebung 1997: $n=217$ ).

\section{Rückkontakt}

Freiwillig erbetene Angaben zur beantwortenden Dienststelle oder Person erfolgten in beachtlicher Höhe von 79 \% der Fälle (gegenüber 1997 mit extrem hoher Quote von 90 \%).

\section{Regionalverteilung}

Am höchsten sind die Teilstichproben vor allem aus Bayern (34\%), gefolgt von NordrheinWestfalen und Baden-Württemberg (je $10 \%$ ), Niedersachsen und Rheinland-Pfalz (9\%), Hessen $(8 \%)$, Schleswig-Holstein (7\%) (keine Vergleichdaten zu 1997).

\section{Regionale Selbstselektion}

Die Analyse beruht auf freiwillig erbetenen Angaben der Postleitregion der beantwortenden Dienststelle (39,2 \% der bearbeiteten Bögen). Als grobe Abschätzung ergibt sich, dass eine quantitativ deutlich verminderte Beteiligung an der Befragung (Chi-Quadrat-PrüfgröBe) aus den PL- Regionen 2 (Hamburg, Schleswig-Holstein) und 5 (Köln) besteht, während die beiden südlichen Schwerpunktregionen 
in Bayern (mit Thüringen) recht klar erhöhte Rücklaufquoten aufweisen (keine Vergleichdaten zu 1997).

\section{Urbanisierungsgrad des Regionalumfeldes}

Innerhalb der Untersuchungsstichprobe findet sich ein deutlicher Schwerpunkt der durchgeführten Radfahrveranstaltungen in dörflichen und ländlichen Gebieten. Gegenüber der Ersterhebung 1997 finden sich dabei nahezu keine Verschiebungen.

\subsection{Umsetzungsrahmen der Radfahrausbildung in Deutschland.}

Die Radfahrausbildung, die meist durch Verkehrserziehungsdienste der Polizei in Kooperation mit Schulen durchgeführt wird, weist derzeit eine nach wie vor sehr hohe Umsetzungsreichweite auf: Gegenüber der Ersterhebung 1997 finden sich keine größeren Verschiebungen. Flächenübergreifend werden 85,7 \% aller Klassen im Rahmen des jeweiligen Zuständigkeitsbereichs der Dienststelle bis zum Abschluss der Grundschule komplett erreicht.

In etwa $23 \%$ der erfassten Regionen liegt die Erstzuständigkeit für die Radfahrausbildung (schwerpunktmäßig) bei der Grundschule, in der weit überwiegenden Mehrzahl der Regionalgebiete 76,7 \% - liegt die vorrangige Zuständigkeit bei Polizeiverkehrslehrern. Dabei zeigen sich quantitativ sehr beträchtliche Unterschiede zwischen den Bundesländern, wobei in Baden-Württemberg, Mecklenburg-Vorpommern, Saarland oder Sachsen ausschließlich, in Bayern, Hessen oder Sachsen-Anhalt ganz überwiegend (jeweils mehr als $85 \%)$ die Rückläufe von Polizeidienststellen stammen, während am anderen Verteilungsende, in Schleswig-Holstein, gerade $8 \%$ der Rückläufe von den Polizeiverkehrslehrern bearbeitet sind.

\section{Lernorte der Ausbildung und Prüfung}

Festzuhalten ist hier eine deutliche Veränderung gegenüber der Ersterhebung 1997: Heute werden in $\mathbf{5 0}$ \% der Regionalgebiete die Radfahrausbildung und -prüfung überwiegend oder zumindest teilweise im realen Verkehrsraum durchgeführt (Ersterhebung 1997: 23,1 \%). Auch hier fällt als regionale Extremposition das Bundesland Schleswig-Holstein auf, in dem mit 91,3\% ein RealraumDurchführungsschwerpunkt berichtet wird (vermutlich gemeinsam mit Hamburg nach den Befunden aus der Ersterhebung (damals $93,3 \%)$ ).

Dennoch bestehen aber auch gegenwärtig noch sehr unterschiedliche Umsetzungsformen; Empfehlungen, bei denen eine bestimmte Form der ortsbezogenen Organisation der Radfahrausbildung und -prüfung als "Sollwert" vorgegeben wird, dürften danach derzeit unverändert keine Aussicht auf generell ausreichende Akzeptanz finden.

\section{Zusatzangebote}

Immerhin $52 \%$ der Befragten beantworten die Frage nach Zusatzangeboten inhaltlich (1997: $18 \%$ der Befragten). Qualitative Angaben beziehen sich vorrangig auf Sehtests.

\subsection{Motorische Schwächen von Kindern}

Es gibt eine deutliche Problemveränderung im Vergleich zu 1997: Nur mehr rund ein Fünftel (23\%) (Ersterhebung: knapp die Hälfte (46,1 \%)) der befragten Verkehrserzieher haben den Eindruck, dass die Anzahl von Kindern, die bei der Radfahrausbildung durch motorische Schwierigkeiten auffallen, etwa gleich geblieben sei. 72 \% (1997: 45,6 \%) nehmen jetzt einen ansteigenden Problemtrend wahr. 
Das heißt, dass die Befunde bezüglich der Verbreitung von motorischen Schwächen der Kinder im Vergleich zum Zeitpunkt der Ersterhebung eine erhebliche, weitere Verdichtung der Problemsituation zum Ausdruck bringen.

\section{Hinweise auf motorische Problemverdichtung} werden weniger für Grundschuleinrichtungen und Schulklassen sondern vielmehr bei bestimmten Gruppen von Kindern gesehen; die Antwortverteilung unterscheidet sich dabei nur wenig von den Einschätzungen der Ersterhebung (Nennungsschwerpunkte: Familien mit Migrationshintergrund, hierbei besonders Mädchen, sozial schwache Familien/Kinder, die kein Fahrrad besitzen, zudem häufig genannt werden überbehütete Kinder, Kinder mit Bewegungsmangel und übergewichtige Kinder.

Insgesamt ist nach der Befundlage davon auszugehen, dass der Umfang bestehender psychomotorischer Schwächen für Kinder aus Großstadtgebieten und aus städtischen Kerngebieten am weitesten fortgeschritten ist.

\section{Auswirkungen auf die Anforderungsbewälti- gung in der Radfahrausbildung}

Die Ergebnisse zeigen, dass sehr grundsätzliche Anforderungen, die beim Radfahren laufend in Alltagssituationen sicher beherrscht werden müssen, bei Kindern mit motorischen Fertigkeitsschwächen nicht zuverlässig bewältigt werden können; dies betrifft generell die Fähigkeit der sicheren Spureinhaltung bei seitlicher oder rückwärtsgewandter Orientierung, vor allem in Kombination mit beabsichtigten Richtungsänderungen und deren Anzeige (auch nach rechts). Auswirkungen von praktischen Fertigkeiten der Radfahrtechnik betreffen demnach nicht seltene Sondersituationen, in denen ein besonders hohes Überforderungsrisiko besteht, sondern vielmehr Grundqualifikationen für die Bewältigung von ständig auftretenden
Teilaufgaben bei der Verkehrsteilnahme. Diese Gesamtbewertung stimmt weitgehend mit den Einschätzungen aus der Ersterhebung von 1997 überein.

\subsection{Organisatorische Maßnahmen zur Sicherung der Kursdurchführung}

\section{Organisatorische Schwierigkeiten}

Immerhin fast $16 \%$ der Kurse erfordern nach den Einschätzungen der Befragten häufig oder fast durchgehend spezielle organisatorische Maßnahmen, die sich aus den Problemen der Fahrfertigkeit von Kindern ergeben.

„Erhebliche“ praktische Schwierigkeiten bei der Ausbildung, die auf motorische Schwächen von Kindern zurückgeführt werden, finden sich in stärker städtisch geprägten Regionen etwas häufiger (Chi-Ou. (9) = 18,1, p < 5 \%).

\section{Konkrete Maßnahmen}

Etwas mehr als $60 \%$ der Befragten berichteten über konkrete organisatorische Maßnahmen, um sich auf das Problem der motorisch schwächeren Kinder in der Radfahrausbildung einzustellen. Mit deutlichem Abstand am häufigsten werden danach derzeit - entweder unmittelbar bei Kursbeginn oder zeitlich früher - Geschicklichkeitsprüfungen zur Radfahrbeherrschung durchgeführt (regelmäßig in 40 \% der einbezogenen Regionalgebiete). Hier zeigt sich keine Veränderung gegenüber der Ersterhebung 1997.

Insgesamt wird derzeit aber auf auftretende motorische Schwächen der Kinder mit organisatorisch deutlich abweichenden und in der Bandbreite erheblich reduzierten Maßnahmen reagiert, als dies noch vor 10 Jahren der Fall war. Vermindert ist vor allem die Zahl der Kurse, in denen regelmäßiger auch Einzelför- 
derungen während des Kurses erfolgt (18\%; 1997: $32 \%)$ sowie das Angebot von Nachkur$\operatorname{sen}(17 \%$; 1997: $33 \%)$.

\subsection{Allgemeinere Problemperspektiven}

\section{(1) Regionale Unfalldichte im Bundesgebiet}

Betrachtet man die regionale Radfahrunfalldichte von Kindern im Bundesgebiet (vgl. [6], S. $26 \mathrm{f}$.), so wird man folgern müssen, dass in den (häufiger in nördlichen Teilregionen gelegenen) Gebieten mit (meist langzeitig stabil) erhöhter Unfallgefährdung die Radfahrausbildung - als Maßnahmefeld der Unfallprävention - nach den Ergebnissen der Studie keineswegs eine stärkere Beachtung als organisatorisches Aufgabenfeld findet. Tendenzmäßig - allerdings mit einigen deutlicheren Abweichungen - zeigt sich vielmehr eine eher gegenläufige Beziehung.

Bedenkenswert erscheint dabei nicht allein die durchschnittlich wohl verminderte Teilnahmequote an der Befragung in den stärker unfallbelasteten Bundesländern, sondern auch, dass drei der vier Bundesländer mit offensichtlich stärker dezentral organisierter - auf die Schulebene verlagerter - Radfahrausbildung durch eine überdurchschnittliche Radfahrunfalldichte von Kindern beschrieben werden müssen (Schleswig-Holstein, Niedersachsen, auch Nordrhein-Westfalen, vgl. Grafik 6).

\section{(2) Motorische Schwächen als Problemursa- che nach der Radfahrausbildung}

Soweit in der aktuellen verkehrswissenschaftlichen Diskussion zur Radfahrsicherheit eine altersbezogene Erweiterung der Radfahrausbildung über den Grundschulbereich hinaus Beachtung findet (vgl. [2]), sollte darauf hinzuweisen sein, dass über ein Fortbestehen von elementaren motorischen Fertigkeiten
- als denkbare Ursache der bekanntlich bis zum Alter von 14 Jahren erheblich zunehmenden Fahrrad-Unfallrisiken von Kindern

- wie auch als einer möglicherweise wichtigen Komponente der Schulwegsicherheit und -planung (vgl. [7])

- sowie schließlich als mögliche Barriere für praktische Ausbildungsteile in diesen anschließenden Altersbereichen derzeit (auch in der internationalen Forschung) gesicherte Erkenntnisse wohl weitestgehend oder völlig fehlen. 


\section{Literatur}

[1] Brandt, K., Eggert, D., Jendritzki, H., \& Küppers, B. (1997). Untersuchungen zur motorischen Entwicklung von Kindern im Grundschulalter in den Jahren 1985 und 1995. Praxis der Psychomotorik, 22 (2), 101 - 107

[2] Deutsche Akademie für Verkehrswissenschaft: 47. Deutscher Verkehrsgerichtstag 2009, Empfehlungen,http://www.deutsche-verkehrsakademie.de/images/stories/pdf/empfehlungen_47vgt.pdf

[3] Ellinghaus, D., Steinbrecher, J.: Radfahrer - Jäger und Gejagte. Eine Untersuchung über die Unfallgefährdung von Radfahrern und der durch sie heraufbeschworenen Gefahren. Aachen: Continental-Uniroyal-Studie 18. 1993

[4] Günther, R.: Psychomotorische Defizite von Kindern im Grundschulalter und ihre Auswirkungen auf die Radfahr-Ausbildung. Ergebnisse einer Befragung der Polizei-Verkehrserziehungsdienste i. A. der deutschen Verkehrswacht Reutlingen/Meckenheim (unveröffentlichter Arbeitsbericht). 1997

[5] Günther, R. und Bergler, M.: Arbeitsplatz stationäre Jugendhilfe. Frankfurt: Internationale Gesellschaft für erzieherrische Hilfen. 1992

[6] Neumann-Opitz, N., Bartz, R., Leipnitz, Ch.: Unfallatlas. Regionale Verteilung von Kinderunfällen in Deutschland. Bergisch-Gladbach: BASt (Mensch und Sicherheit Heft M 192). 2008

[7] BASt-Forschungsprogramm, Projekt FE 82.322/2007

Deutsche Verkehrswacht: Die Radfahrausbildung als integrierter Teil der Verkehrserziehung in der Schule. Meckenheim (2. Auflage). 1991

Deutsche Verkehrswacht: Lehrerhandbuch Radfahren in der Schule. Bonn (4. überarbeitete Auflage). 2007 


\section{Fragebogen zur Radfahrausbildung in der Grundschule}

An die Kolleg/-innen der Polizei- bzw. Verkehrserziehungsfachberater (hier evtl. eine kurze Einleitung)

Bitte faxen Sie den ausgefüllten Fragebogen bis spätestens

an Fax: 0228 / $43380-50$ Deutsche Verkehrswacht, Alexanderstraße 10, 53111 Bonn Herzlichen Dank für Ihre Unterstützung!

1. Die Radfahrausbildung und -prüfung wurde von unserer Dienststelle im vergangenen Schuljahr in etwa Damit wurden in unserem Zuständigkeitsbereich (ca.) ......... Grundschulklassen durchgeführt. Abschluss der Grundschule erreicht. In die Ausbildung einbezogen waren im letzten Schuljahr insgesamt ca. ........ Kinder. Unsere Dienststelle gehört zum Bundesland

2. Was sind Ihre allgemeinen Erfahrungen: Für die Durchführung der Radfahrausbildung ergeben sich durch mangelhafte Körperbeherrschung und verminderte motorische Fertigkeiten beim Radfahren von bestimmten Kindern
( ) keine Probleme
( ) geringe Probleme
( ) teilweise größere Probleme
( ) regelmäßig zu erheblichen Problemen

3. Meiner/ unsere Einschätzung zum Trendverlauf in den zurückliegenden Jahren: Die Zahl von Kindern, die bei der Radfahrausbildung durch mangelhafte Körperbeherrschung /Radfahrfertigkeiten auffallen,
( ) hat erheblich abgenommen
( ) hat leicht abgenommen
( ) ist etwa gleich geblieben
( ) hat leicht zugenommen
() hat erheblich zugenommen

4. Wenn motorische Schwächen bei bestimmten Kindern auftauchen: Bei welchen Ausbildungsteilen/ Fahraufgaben der Ausbildung führt dies besonders häufig/deutlich zu Schwierigkeiten im Verhalten? (Bitte kurz erläutern)

5. Falls Ihnen ein Ausbildungskurs in Erinnerung ist, bei dem durch Kinder mit Bewegungsmängeln Auswirkungen auf die Kursdurchführung besonders deutlich waren, wäre eine kurze, plastische Beschreibung der Schwierigkeiten für uns hilfreich: 


\section{Fragebogen zur Radfahrausbildung in der Grundschule}

6. Können Sie nach Ihren Erfahrungen ungefähr abschätzen, wie hoch der Anteil der Schulklassen liegt, in denen

kein Kind deutliche Geschicklichkeits- und Fertigkeitsbeeinträchtigungen aufweist

$$
\begin{array}{lll}
5 \text { und mehr Kinder der Klasse deutliche Beeinträchtigungen aufweisen } & \text { ca. } & \ldots . . . \% \text { der Schulklassen } \\
\text { ca. } & \text {..... \% der Schulklassen }
\end{array}
$$

7. Führen oder führten Sie sonstige Untersuchungen (z.B. Sehtests) mit Kindern in Zusammenhang mit der Radfahrausbildung durch? Ggf. auch: Warum jetzt nicht mehr?

8. Führen motorische Defizite von Kindern zu so erheblichen Schwierigkeiten bei der Durchführung von fahrpraktischen Ausbildungskursen, dass Sie sich durch organisatorische Maßnahmen darauf einstellen müssen?
( ) nein, bisher nicht/kaum
( ) ja, gelegentlich
( ) ja, häufig
( ) ja, fast bei jedem

Kurs

Falls ja - wie versuchen Sie sich darauf einzustellen?

Diagnose: Geschicklichkeitsprüfung zur Radfahrbeherrschung bei/vor Kursbeginn.................. vorbereitender Förderkurs

für alle Kinder der Klasse..................... ()

für Kinder mit speziellen Schwächen....... ()

Meist/immer gelegentl., nein/bei Bedarf

intensivere Einzelförderung während des Ausbildung mit der Schulklasse..................................... ()

Zeitliches Zurückstellen (Kind soll zu späterem Zeitpunkt an Ausbildung teilnehmen)........... ( )

Nachkurse für Kinder m.besond. motor. Schwächen
(z.B. gemeinsam mit "Durchgefallenen").... ( ) ( )

Sonstige Maßnahmen, Angebote (bitte kurz beschreiben)

( )

)

()

( )

( )

( )

( )

( )

( )
( )

() 


\section{Fragebogen zur Radfahrausbildung in der Grundschule}

9. Haben Sie den Eindruck, daß Geschicklichkeitsdefizite unterschiedlich häufig auftreten:

In bestimmten Grundschuleinrichtungen (ohne Sonderschulen)
( ) nein
( ) ja. Vielleicht zu erklären durch:

In bestimmten Schulklassen (ohne Förderklassen)
( ) nein
( ) ja. Vielleicht zu erklären durch:

Bei bestimmten Kindern
( ) nein
( ) ja. Nach unserer Beobachtung häufiger bei:

10. Führen Sie die praktische Fahrausbildung (teilweise) im realen Verkehrsraum durch? (Mehrfachankreuzung möglich, wenn örtlich oder situativ unterschiedlich):

( ) nein, nur in der stationären oder mobilen JVS)

( ) Teile des Ausbildungsprogramms in der JVS, Teile im Realraum

( ) Ausbildungsprogramm ganz oder größtenteils im Realraum

Bei Mehrfachankreuzung: Ihre häufigste Durchführungsform bitte durch XX markieren

11. Sonstige Anmerkungen, Hinweise, z.B. Besonderheiten der Kursdurchführung bei Ihnen

12. Unser Einzugsgebiet für die Radfahrausbildung umfaßt ungefähr in \% folgende Gebiete:
..... \% Großstadtisch
...... \% Städtisch
...... \% Kleinstädtisch
..... \% Ländlich.

Noch eine Bitte: Für ggf. kurze Rückfragen (z.B., falls Ihr Fragebogen besonders interessante Hinweise enthält), wären wir für Angabe zu telefon. Kontaktmöglichkeit dankbar: 


\section{GDV}

DIE DEUTSCHEN VERSICHERER

\section{Gesamtverband der Deutschen Versicherungswirtschaft e. V.}

Wilhelmstraße 43 / 43 G, 10117 Berlin

Postfach 0802 64, 1002 Berlin

Tel. 030 / 2020 -50 00, Fax 030 / 2020 - 6000

www.gdv.de, www.udv.de 